\title{
Hybrid zones and voter model interfaces
}

\author{
J.T. $\operatorname{COX}^{1}$ and R. DURRET T ${ }^{2}$ \\ ${ }^{t}$ Department of Mathematics, Syracuse University, Syracuse NY 13210, USA \\ ${ }^{2}$ Department of Mathematics, White Hall, Cornell University, Ithaca NY 14853, USA
}

We study the dynamics of hybrid zones in the absence of selection. In dimensions $d>1$ the width of the hybrid zone grows as $\sqrt{t}$ but in one dimension the width converges to a non-degenerate limit. We believe that tight interfaces are common in one-dimensional particle systems.

Keywords: Hybrid zones; voter modei interfaces; random walk; recurrent potential kernel; stochastic spatial model

\section{Introduction}

In a number of situations one finds two regions in space which contain relatively homogeneous populations, that differ considerably from each other, and are separated by a narrow zone in which hybrids are found. A textbook example is the common house mouse in Denmark (see Hunt and Selander 1973) which exists in the form Mus musculus in the North and in the form $M$. domesticus in the South and along parts of the western coast. A second example is the Northern flicker in the United States (Moore and Koenig 1986), which is red in the West and yellow in the East, with the transition occurring sharply in the western half of South Dakota, Nebraska and Kansas.

There are literally dozens of such examples - see Harrison (1990) for a survey. These zones are of particular interest since they reveal the interaction between divergent genotypes, and may have some bearing on the mechanism of speciation. There are a number of possible explanations for hybrid zones. They might be due simply to a recent mixing of two different types. There might be a sharp ecotone (environmental gradient) so that different types are favoured on opposite sides. A third possibility, called a 'tension zone', occurs when hybrids are less fit.

To be able to distinguish between the three possible causes one needs a model that predicts the evolution of the hybrid zone in the three scenarios. Here we will formulate a model for the evolution of a tension zone. To start with the simplest possible case, we will suppose that only a single locus is involved, and consider a process in which the state at time $t$ is $\eta_{t}: \mathbb{Z}^{d} \times\{\mathbf{I}, 2\} \rightarrow\{\mathrm{A}, \mathrm{a}\}$, where $\left(\eta_{l}(x, 1), \eta_{l}(x, 2)\right)$ gives the state of the individual at $x$ at time $t$. To formulate the dynamics we let $\phi_{\mathrm{AA}}, \phi_{\mathrm{Aa}}=\phi_{\mathrm{aA}}$ and $\phi_{\mathrm{aa}}$, all $\in[0,1]$, be the relative fitnesses of the three types of individuals. At rate 1 , each individual is replaced by a new individual. To make a new individual at $x$, we first choose one parent according to $p(x, y)$, pick one of its two genes at random, then choose a second parent independently according to $p(x, y)$, and pick one of its two genes. Combining the two chosen genes we get the type, say $i j$, of the proposed new individual. We generate a random variable $U$ that is uniform on $(0,1)$ and accept this individual if $\phi_{i j}<U$. If the proposed new individual is rejected, we pick new parents and repeat the process again using new choices that are independent of the old ones. 
People who have moral objections to our homosexual mating can enlarge each site to be a 'deme' consisting of $M$ males and $N$ females, rewrite the state as $\eta_{t}: \mathbb{Z}^{d} \times\{1, \ldots, 2(M+N)\}$, regard the first $2 M$ locations as being male genes, and pick one male and one female parent. Such a generalization is important if one is investigating sex-linked characteristics, as in Silver's study (in preparation) of thaplotypes in mice. However, in this paper we will concentrate on the behaviour of the asexual model with one individual per site and no selection (i.e. $\phi_{i j} \equiv 1$ ), since this system is closely related to a voter model on $\mathbb{Z}^{d} \times\{1,2\}$. While this is not the case of most interest to biologists, it is none the less useful to know the behaviour of the system under the null hypothesis of no selection - for example, in order to reject this hypothesis and conclude that the effect of selection is significant.

To prove results we need assumptions about the parental choice matrix $p$. The first and most basic are the following:

(i) $p$ is translation invariant, that is, $p(x, y)=q(y-x)$.

(ii) $p$ is irreducible, that is, it is possible to get from 0 to any $x \in \mathbb{Z}^{d}$ in a finite number of steps.

(iii) $q$ has finite second moments, that is, $\sum_{x}|x|^{2} q(x)<\infty$.

For simplicity we will also assume

(iv) $p$ is symmetric, that is, $p(x, y)=p(y, x)$ or $q(-z)=q(z)$.

The last assumption seems natural from a biological point of view and makes the drift $\mu=\sum_{x} x q(x)=0$. To treat the general case one has only to subtract the drift and introduce the symmetrized kernel

$$
\tilde{p}(x, y)=\frac{p(x, y)+p(x,-y)}{2}
$$

at the appropriate places.

To formulate our first result we will replace $A$ by 1 and a by 0 to return to a more traditional notation of particle systems, and we will consider an initial configuration in which

$$
\eta_{0}(x)= \begin{cases}(0,0) & \text { if } x_{1} \geq 0 \\ (1,1) & \text { if } x_{1}<0\end{cases}
$$

For $x \in \mathbb{Z}^{d}$ let $\theta_{x}$ be the operator which shifts the configuration of the process so that the site $x$ sits at the origin. Let

$$
\Gamma_{i j}=\sum_{x} x_{i} x_{j} q(x) \quad \sigma_{i}^{2}=\Gamma_{i i}
$$

be the covariance matrix of $q$ and the variance of the ith component, which is positive by (ii), and let $\Phi(x)$ be the standard normal distribution function. Finally, let $\nu_{\rho}$ be the limiting state for our model starting from product measure with density $\rho$, that is, the genes in the initial configuration are independent and are equal to 1 with probability $\rho$. Results of Holley and Liggett (1975) imply that $\nu_{\rho}$ is a non-trivial stationary distribution in $d \geq 3$, while in $d \leq 2, \nu_{\rho}=\rho \delta_{1}+(1-\rho) \delta_{0}$ where $\delta_{i}$ is the point mass of the $\equiv i$ configuration.

With all this notation introduced we can state our first result simply as follows: 
Theorem 1 Suppose $x_{t} \in \mathbb{Z}^{d}$ and $x_{t} / \sqrt{t} \rightarrow x$ as $t \rightarrow \infty$. Then

$$
\theta_{x_{t}} \eta_{t} \Rightarrow \nu_{\Phi}\left(-x_{1} / \sigma_{1}\right) \text {. }
$$

Presutti and Spohn (1983) have proved a closely related hydrodynamic limit which treats a general sequence of initial conditions. However, except for a remark on p. 870 , their investigation is restricted to $d \geq 3$; and they suppose that the density of $1 \mathrm{~s}$ in the initial configuration is asymptotically a continuous function of $x$.

In the biologically interesting cases $d \leq 2$, Theorem 1 does not tell us much about the structure of the 'hybrid zone'. Our next result shows that, in $d=2$, if we paint 1 s white and 0 s black then the normal distribution predicts the shade of grey we see.

Theorem 2 In $d=2$, if $h$ is a continuous function with compact support, then as $t \rightarrow \infty$

$$
\frac{1}{2 t} \sum_{x \in \mathbb{Z}^{2}} \sum_{i=1}^{2} \eta_{t}(x, i) h(x / \sqrt{t}) \rightarrow \int h(y) \Phi\left(-y_{1} / \sigma_{1}\right) \mathrm{d} y \quad \text { in probability. }
$$

The next result deals with the density of heterozygotes in the hybrid zone.

Theorem 3 In $d=2$, if $x_{t} \in \mathbb{Z}^{2}$ and $x_{t} / \sqrt{t} \rightarrow x$ as $t \rightarrow \infty$, then

$$
P\left(\eta_{t}\left(x_{t}, 1\right) \neq \eta_{t}\left(x_{t}, 2\right)\right) \sim 2 \Phi\left(-x_{1} / \sigma_{1}\right)\left\{1-\Phi\left(-x_{1} / \sigma_{1}\right)\right\} \frac{C}{\log t} .
$$

Theorem 3 is easy to see since (i) there is a duality between the voter model and coalescing random walks that says that the probability $\eta_{t}\left(x_{i}, 1\right) \neq \eta_{t}\left(x_{t}, 2\right)$ is the probability that two random walks starting at $(x, 1)$ and $(x, 2)$ do not hit by time $t$; (ii) results for random walks say that this probability is asymptotically equal to $C / \log t$; and (iii) if we condition two two-dimensional random walks not to hit by time $t$, when divided by $\sqrt{t}$ they converge to two independent normals. We can see (iii) using ideas of Durrett (1978) by noting that two-dimensional Brownian motion does not hit 0 , so conditioning Brownian motion to avoid 0 has no effect.

While the last paragraph should make Theorem 3 plausible to mathematicians, Theorem 3 is disturbing to geneticists since it contradicts the Hardy-Weinberg equilibrium, which predicts that when the allele A has frequency $p$ the genotypes AA, Aa and aa are always found in frequencies $p^{2}$, $2 p(1-p)$ and $(1-p)^{2}$, respectively. There is no logical contradiction, since the Hardy-Weinberg equilibrium is derived in a non-spatial model and it is easy to see from duality and the recurrence of two-dimensional random walk that it cannot hold here. However, the failure of Hardy-Weinberg equilibrium in our model is disturbing since it is widely observed to hold in nature.

To resolve this 'paradox' we have simulated the model with $p(x, y)=1 / 25$ when $\sup _{i}\left|x_{i}-y_{i}\right| \leq 2$ in a $120 \times 120$ universe with cylindrical boundary conditions: the top and bottom of the square are identified but not the left and right edges. Figure 1 shows the average of the states of five runs at time 250 starting with all AAs at points with $x \geq 61$ and all aas at points with $x \leq 60$. Here we have plotted the average fraction of AAs and Aas in each column $x=c$. The graph of the fraction of AAs approximates the normal distribution function as predicted by Theorem 2 . Note, however, that 
Time $=250$

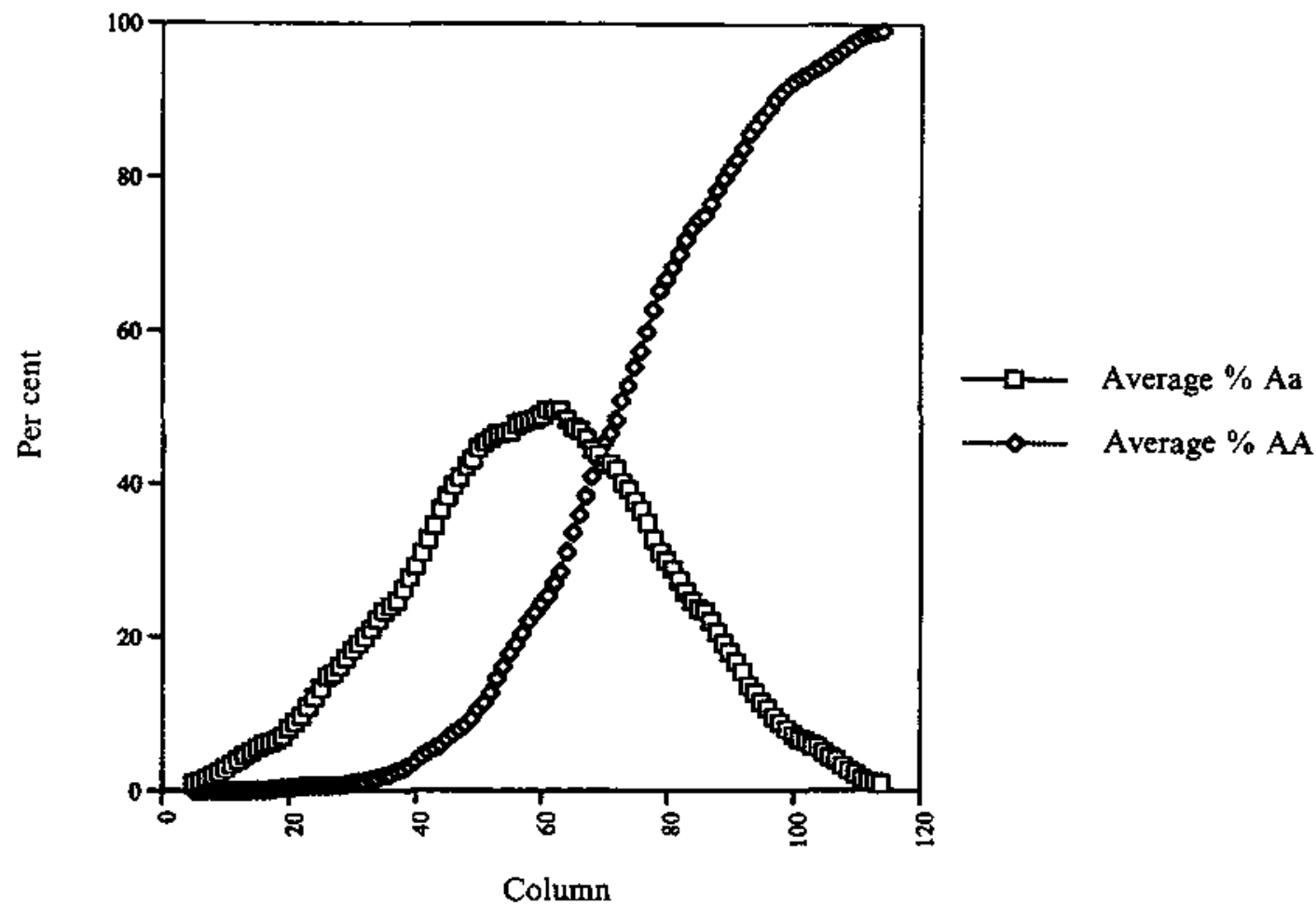

Figure 1.

the graph of the fraction of Aas is much closer to what we would expect if each column was in Hardy-Weinberg equilibrium than to the $\equiv 0$ limit predicted by Theorem 3 . This resolves the paradox since it indicates that over 'short' time-scales (here roughly 250 generations) there is little deviation from Hardy-Weinberg equilibrium, while the clustering predicted in Theorem 3 takes place over tens or hundreds of thousands of years.

Last and most interesting is the case of one dimension. Since the proofs here are more involved, we will consider the voter model on $\mathbb{Z}$ instead of on $\mathbb{Z} \times\{1,2\}$. Based on the proofs of Theorems 1-3 we expect that the result will stay the same, and simulations we have done confirm this, but the proof will be much longer since we have to prove results for random walks on $\mathbb{Z} \times\{1,2\}$ that are the analogues of classical results for random walks on $\mathbb{Z}$. We ask the reader to postpone judging our sin until after reading all the details in Sections 3 and 4.

For the voter model on $\mathbb{Z}$ we define the leftmost-zero and the rightmost-one processes

$$
\ell_{t}=\inf \left\{x: \eta_{t}(x)=0\right\} \quad r_{t}=\sup \left\{x: \eta_{t}(x)=1\right\}
$$

and the interface process $\iota_{t}$

$$
\iota_{t}(x)=\eta_{t}\left(x+\ell_{t}-1\right), \quad x \geq 0 .
$$


The process $\iota_{t}$ takes values in the countable set

$$
\Xi_{0}=\left\{\xi: \mathbb{Z}^{+} \rightarrow\{0,1\}, \xi(0)=1, \xi(1)=0, \sum_{x \geq 0} \xi(x)<\infty\right\}
$$

For $\xi \in \Xi_{0}$ let $r(\xi)=\sup \{x: \xi(x)=1\}$. For the one-dimensional results we have to assume in addition to (i)-(iv) that

(v) $\sum_{z}|z|^{3} q(z)<\infty$.

We need this to know that the 'ladder variables' of the random walk have finite second moments and hence the limiting overshoot distribution has finite mean - see the proof of Lemma 3. Of course, if one wants to remove (v), one should also ask if recurrence of the random walk (or more simply finite mean) is enough for the next result.

Theorem 4 The interface process $\iota_{t}$ is an irreducible, positive recurrent Markov chain. So there is a probability $\pi$ on $\Xi_{0}$ such that

$$
P\left(\iota_{t}=\xi\right) \rightarrow \pi(\xi) \quad \text { as } t \rightarrow \infty, \xi \in \Xi_{0}
$$

Consequently,

$$
P\left(r_{t}-\ell_{t}=k\right) \rightarrow \pi(\{\xi: r(\xi)=k+1\}) \quad \text { as } t \rightarrow \infty .
$$

If $x_{t} / \sigma \sqrt{t} \rightarrow r$, it follows easily from the duality between the voter model and coalescing random walks that $P\left(\eta_{t}\left(x_{t}\right)=1\right) \rightarrow \Phi(-r)$. Since Theorem 4 implies $P\left(\eta_{t}\left(x_{t}\right)=1\right) \approx P\left(r_{t} \geq x_{t}\right)$ it follows almost immediately that we have:

Theorem 5 Assume $\sigma^{2}>0$. Then

$$
P\left(r_{t} / \sigma t^{1 / 2} \leq x\right) \rightarrow \Phi(x) \quad \text { as } t \rightarrow \infty
$$

Of course, the last conclusion also holds for $\ell_{t}$. Combining Theorems 4 and 5 with our belief that the models on $\mathbb{Z}$ and $\mathbb{Z} \times\{1,2\}$ exhibit similar behaviour gives the following mental picture for hybrid zones in $d=1$ : all hybrids are confined to an interval $\left[\ell_{t}, r_{t}\right]$ that has length $O(1)$ and its midpoint at $\approx \chi \sigma \sqrt{t}$ when $\chi$ has the standard normal distribution.

One way of proving Theorem 4 would be to show that $E\left(r_{t}-\ell_{t}\right)$ stays bounded. We first believed that this is false.

Guess 1 Let $\bar{\pi}_{k}=\pi(\{\xi: r(\xi)=k+1\})$. Then $\sum_{j \geq k} \bar{\pi}_{j} \sim C / k$ as $k \rightarrow \infty$.

Our intuition here was that there is a probability of the order of $1 / \sqrt{t}$ that two random walks starting at $-\sqrt{t}$ and $\sqrt{t}$ will cross without hitting and end up on the opposite side of 0 at time $t$. Of course, what this implies is that $P\left(r_{t}-t \geq \sqrt{t}\right) \geq C / \sqrt{t}$, and we need to let the first $t \rightarrow \infty$ to talk about the limit distribution.

To investigate this guess, we simulated the discrete-time voter model with $p(x, y)=\frac{1}{7}$ for $|x-y| \leq 3$. Running the process out to time 100000 and keeping track of the width of the 


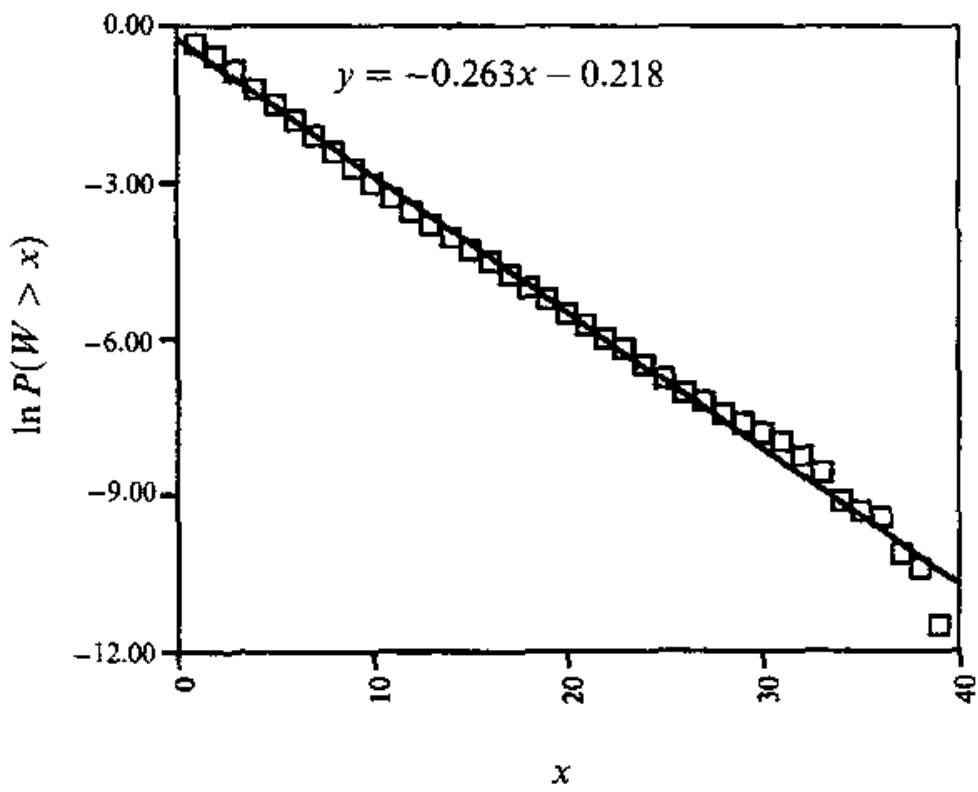

Figure 2.

interface at each time gave us an estimate of the distribution of the width in equilibrium, $\bar{\pi}_{k}=P(W=k)$. We then plotted $\ln P(W>x)$ versus $\ln x$ to estimate the polynomial rate at which $P(X>x)$ went to 0 but this gave us a curve, so we plotted $\ln P(W>x)$ versus $x$ and got a nice straight line (see Fig. 2). This led to

Guess $2 \quad \bar{\pi}_{k} \leq C \mathrm{e}^{-\uparrow k}$.

After a lot of heip we now know the following:

Theorem 6 If we exclude the nearest neighbour case in which the interface $t_{t}=\iota_{0}$ for all $t$ then $\sum_{k} k \bar{\pi}_{k}=\infty$, that is, the equilibrium interface distribution has infinite mean.

The idea for this proof comes from joint work of Carl Mueller and Roger Tribe, which Roger described to Ted Cox at the Bernoulli Society World Congress at Chapel Hill, NC, 1994. However, to investigate the details we needed the foliowing result which was proved by Greg Lawler and Harry Kesten. Let $S_{n}=X_{1}+\ldots+X_{n}$ be a one-dimensional aperiodic random walk. Let

$$
a(x)=\sum_{m=0}^{\infty} P^{0}\left(S_{m}=0\right)-P^{0}\left(S_{m}=x\right)
$$

be the recurrent potential kernel. Let $\tau_{0}=\inf \left\{n: S_{n} \leq 0\right\}$. 
Theorem 7 If $\mathrm{E} X_{i}=0$ and $\mathrm{E} X_{i}^{2}=\sigma^{2} \in(0, \infty)$ then, for $x \geq 0$,

$$
a(x)=x / \sigma^{2}+\mathrm{E}^{x}\left\{\left(S_{\tau_{0}} / \sigma^{2}\right)+a\left(S_{\tau_{0}}\right)\right\}
$$

and hence if $\mathrm{E}\left|X_{i}\right|^{3}<\infty$ then $a(x)=x / \sigma^{2}+\alpha+o(1)$.

The second term and the constant $\alpha$ vanish for nearest neighbour random walk but are strictly positive for other cases, and this is the key to the proof of Theorem 6 . Readers who find this result more interesting than the rest of the paper can go now to the beginning of Section 3 and read its proof.

In the rest of this section we discuss interfaces in other models. We believe that the phenomenon of tight interfaces is common in one-dimensional particle systems. Consider the contact process on $\mathbb{Z}$. In this model the state at time $t$ is $\eta_{t}: \mathbb{Z} \rightarrow\{0,1\}$ with 0 standing for 'vacant' and 1 for 'occupied', and the system evolves as follows:

(a) Occupied sites become vacant at rate 1 .

(b) If $x$ is vacant, it becomes occupied at rate $\beta \sum_{y} p(x, y) \eta_{k}(y)$.

As above, $p(x, y)=q(y-x), p$ is irreducible and symmetric, but this time we are willing to assume in addition that $q$ has finite range, that is, $q(z) \neq 0$ for only finitely many $z$.

Let $\xi_{i}^{+}$be the contact process starting from $\xi_{0}^{+}(x)=1$ when $x \leq 0$, and 0 otherwise. Let $\xi_{i}^{1}$ be the contact process starting from $\xi_{0}^{l}(x) \equiv 1$ and suppose that the two processes are constructed on the same space using the graphical representation of Harris (1978) and Griffeath (1979). Let

$$
\begin{aligned}
& r_{t}=\sup \left\{x: \xi_{t}^{+}(x)=1\right\} \\
& \ell_{t}=\inf \left\{x: \xi_{t}^{+}(x)=0, \xi_{t}^{1}(x)=1\right\} .
\end{aligned}
$$

Since $\xi_{t}^{+}(x) \leq \xi_{t}^{l}(x), \ell_{t}$ is the location of the leftmost discrepancy. We refrain from defining the interface process $\iota_{y}$ since it is no longer a countable-state Markov chain and make the following rather weak conjecture:

Conjecture 1 If $\beta>\beta_{\mathrm{c}}$ then the sequence $\left\{r_{t}-\ell_{t}, t \geq 0\right\}$ is stochastically compact.

Here $\beta_{\mathrm{c}}$ is the critical value for survival of the contact process - see, for example, Liggett (1985, Chapter VI) or Durrett (1988, Chapters 4 and 11). One should be able to prove this by combining results of Bezuidenhout and Grimmett (1990) with a block argument (see Durrett 1993 for a number of applications of this technique) but we have not been able to cope with the details.

A much more exciting interface problem to attack is the one-dimensional version of Ziff et al.'s (1986) model of oxidation of carbon monoxide on a catalyst surface. In this process the state at time $t$ is $\eta_{t}: \mathbb{Z} \rightarrow\{0,1,2\}$ where 0 stands for a vacant site, while 1 and 2 , respectively, indicate a carbon monoxide $(\mathrm{CO})$ molecule or oxygen $(\mathrm{O})$ atom attached to the surface. The system evolves as follows:

(a) Carbon monoxide molecules land at vacant sites at rate $p$.

(b) An adjacent pair of vacant sites becomes occupied by two oxygen atoms at rate $q / 2$.

(c) Adjacent $\mathrm{CO}$ and $\mathrm{O}$ atoms react at rate $r \in(0, \infty]$, producing two adjacent vacant sites. 
In the case $r=\infty$, which is the original Ziff et al. model, the dynamics are defined by letting $r \rightarrow \infty$. That is, each newly landed $\mathrm{O}$ atom or $\mathrm{CO}$ molecule checks its neighbours to see if a reaction can occur. If so, a reaction occurs immediately and two vacant sites result. If a $\mathrm{CO}$ molecule lands between two $\mathrm{O}$ atoms it reacts with one of them chosen at random.

Consider the Ziff et al. model starting from $\eta_{0}(x)=1$ for $x \leq 0$ and $\eta_{0}(x)=2$ for $x>0$, and let

$$
\begin{aligned}
\ell_{t} & =\inf \left\{x: \eta_{t}(x)=2\right\} \\
r_{t} & =\sup \left\{x: \eta_{t}(x)=1\right\} \\
\iota_{t}(x) & =\eta_{t}\left(x+\ell_{t}-1\right), \quad x \geq 0 .
\end{aligned}
$$

Conjecture 2 The conclusions of Theorem 4 hold, and there are constants $m=m(p)$ and $\sigma$ such that

$$
P\left(\frac{r_{1}-m t}{\sigma t^{1 / 2}} \leq x\right) \rightarrow \Phi(x) .
$$

The interest in this result is that it is an important first step in showing that coexistence is not possible in $d=1$. For example:

Conjecture 3 Consider the system with fixed $q$ and $r$. The 'edge speed' $m(p)$ is a strictly increasing function of $p$ and hence has $m\left(p_{0}\right)=0$ for only one value of $p$. If we consider an initial state in which there are infinitely many $1 \mathrm{~s}$ and $2 \mathrm{~s}$ but no $1 \mathrm{~s}$ adjacent to $2 \mathrm{~s}$ then

$$
\xi_{t} \Rightarrow \begin{cases}\delta_{1} & \text { when } p>p_{0} \\ \delta_{2} & \text { when } p<p_{0}\end{cases}
$$

Ziff et al. (1986) used computer simulations to demonstrate that in $d=2$ when $r=\infty$ and $q=1-p$ there are two critical values $p_{1} \approx 0.389$ and $p_{2} \approx 0.525$ so that when $p_{1}<p<p_{2}$ coexistence occurs, that is, there is a non-trivial stationary distribution in which $1 \mathrm{~s}$ and $2 \mathrm{~s}$ are present at positive density, while for $p<p_{1}$ or $p>p_{2}$ we have convergence to $\delta_{2}$ or $\delta_{1}$, respectively. It is not hard to prove that the last two conclusions hold for small $p$ and $p$ close to 1, respectively, but proving coexistence for the original Ziff $e t$ al. model appears to be a difficult problem. Durrett and Swindle (1993) and Bramson and Neuhauser (1992) have proved coexistence results for modified versions of the Ziff et al. model.

To complete our survey of the literature, we should mention the simple exclusion process. In this model particles on $\mathbb{Z}$ independently jump one unit to the right at rate $p>1 / 2$ and one unit to the right at rate $1-p$ subject to the exclusion rule: jumps onto occupied sites are not allowed. This process has a tight interface in the following sense. There is a measure $\mu$ on the space of configurations approaching asymptotically the product measure with densities $\rho<\lambda$ to the left and the right of the origin respectively, and a random position $X(t)$ such that if we start from $\mu$ at time 0 then the system as seen from $X(t)$ remains distributed according to $\mu$ for all $t \geq 0$. This description is from Ferrari et al. (1991). Their paper can be consulted for details and references to earlier work on this problem. This picture has been developed further by Ferrari (1992) and Ferrari and Ravishankar (1992). 
Finally, in view of Tribe's help with Theorem 6, we should mention Tribe (1993) and Mueller and Sowers (1994) where related problems for the stochastic partial differential equations are studied. The rest of this paper is devoted to the results that we do know how to prove. The first three theorems are quite easy and are shown in Section 2. In Section 3 we prove the random walk results (for example, Theorem 7) that are the keys to the proofs of Theorems 4-6 given in Section 4. Finally, in the Appendix we prove a limit theorem for one-dimensional random walk conditioned to avoid 0 that is needed in the proof of Lemma 3 . We give the proof because (i) the result is stated incorrectly in Belkin (1970); (ii) it is short; and (iii) we need to invert the characteristic function that appears in the limit.

\section{Proofs of Theorems 1-3}

Our first step is to introduce the duality between the voter model and coalescing random walks, using a slightly non-standard notation that we find simpler in this case than the traditional 'arrowdelta' approach - see Griffeath (1979) or Durrett $(1988 ; 1993)$. For each $x \in \mathbb{Z}^{d}$, let $\left\{T_{n}^{x}, n \geq 1\right\}$ be a Poisson process with rate 1 and for $i=1,2$ let $\left\{Y_{n}^{x, i}, n \geq 1\right\}$ and $\left\{J_{n}^{x, i}, n \geq 1\right\}$ be independent i.i.d. sequences with $P\left(Y_{n}^{x, i}=y\right)=p(x, y)$ and $P\left(J_{n}^{x, i}=j\right)=1 / 2$ for $j=1,2$. At time $T_{n}^{x}$ we draw an arrow from $(x, 1)$ to $\left(Y_{n}^{x, 1}, J_{n}^{x, 1}\right)$ and from $(x, 2)$ to $\left(Y_{n}^{x, 2}, J_{n}^{x, 2}\right)$ to indicate where the new genetic material comes from.

Even though there are infinitely many Poisson processes and hence no first arrival, it is easy to adapt an argument of Harris (1972) to show that our recipe allows us to construct our process $\eta_{t}$. For details, see Durrett (1993, Section 2). The reason for using this construction is that it allows us to work backwards in time. For each $(x, i) \in \mathbb{Z}^{d} \times\{1,2\}$ and $t \geq 0$ we define a process $S_{s}^{(x, i), t} \in \mathbb{Z}^{d} \times\{1,2\}, 0 \leq s \leq t$, that 'follows the arrows'. That is, if the tail of an arrow touches $S_{s}^{(x, i), t}$ at time $t-s$, then $S_{s}^{(x, i), t}$ jumps to the head of the arrow. It is easy to see that $S_{s}^{(x, i), t}$ is a random walk, and that the state of $(x, i)$ at time $t$ is the same as that of $S_{s}^{(x, i), t}$ at time $t-s$.

We define the dual process by

$$
\xi_{s}^{A, t}=\left\{S_{s}^{(x, i), t}:(x, i) \in A\right\}
$$

for each $A \subset \mathbb{Z}^{d} \times\{1,2\}$. From the definition of the $S_{s}^{(x, i), z}$, it is clear that

$$
P\left(\eta_{t}(z) \equiv 0 \text { on } A\right)=P\left(\eta_{0}(z) \equiv 0 \text { on } \xi_{t}^{A, t}\right)
$$

This is the duality equation. The process $\xi_{t}$ is called a coalescing random walk since if $S_{s}^{(x, i), t}=S_{s}^{(y, i), t}$ we will have $S_{r}^{(x, i), t}=S_{r}^{(y, i), t}$ for all $r \geq s$. Note also that the two processes $S_{s}^{(x, i), t}$ and $S_{s}^{\left(y_{r}, i\right), t}$ move independently as long as their $\mathbb{Z}^{d}$ coordinates are not equal.

\section{Proof of Theorem 1}

Suppose, first, that $d \geq 3$. Let $F \subset \mathbb{Z}^{d} \times\{1,2\}$ be a finite set and consider a coalescing random walk $\xi_{s}$ starting with particles on $\left(x_{t}, 0\right)+F$. To estimate the behaviour of $\xi_{s}$, we introduce a comparison process $\zeta_{s}$ which behaves like $\xi_{s}$ until time $\sqrt{t}$ and then allows the surviving particles to move like independent random walks. It is easy to see that the probability that two particles in $\xi_{s}$ collide between times $\sqrt{t}$ and $t$ goes to 0 as $t \rightarrow \infty$ so the probability $\xi_{t}=\zeta_{t}$ converges to 1 . 
To estimate the behaviour of $\xi$, we observe that the central limit theorem implies that when the number of particles at time $\sqrt{t},\left|\xi_{\sqrt{t}}\right|=k$, the $k$ particles are all within $t^{1 / 3}=(\sqrt{t})^{2 / 3}$ of $x_{t}$ with high probability. Now, from time $\sqrt{t}$ to time $t$ the $\mathbb{Z}^{d}$ components of the particles in $\xi_{s}$ move like independent copies of a random walk $R_{t}$ that takes jumps distributed according to $q$ at rate 1 , so if we let $R_{s}^{(1)}$ denote the first component of $R_{s}$ it follows from the central limit theorem that

$$
\sup _{y:|y| \leq t^{1 / 3}}\left|P\left(R_{t-\sqrt{t}}^{(1)} \leq 0 \mid R_{0}=x_{t}+y\right)-\Phi\left(-x_{1} / \sigma_{1}\right)\right| \rightarrow 0 .
$$

The reader should note the little parentheses around the 1. In many cases below we will use plain superscripts to denote different copies of a multidimensional process.

Let $\eta_{t}^{p}$ denote the voter model starting from product measure with density $p$. Combining the results in the last two paragraphs with the duality equation, it follows that

$$
\begin{aligned}
P\left(\eta_{t} \equiv 0 \text { on }\left(x_{t}, 0\right)+F\right) & =P\left(\eta_{0} \equiv 0 \text { on } \xi_{t}^{\left(x_{t}, 0\right)+F, t}\right) \\
& \approx P\left(\eta_{0}^{\Phi\left(-x_{1} / \sigma_{1}\right)} \equiv 0 \text { on } \xi_{\sqrt{t}}^{\left(x_{t}, 0\right)+F, t}\right) \\
& =P\left(\eta_{0}^{\Phi\left(-x_{1} / \sigma_{1}\right)} \equiv 0 \text { on } \xi_{\sqrt{t}}^{F, \sqrt{t}}\right) \\
& =P\left(\eta_{\sqrt{t}}^{\Phi\left(-x_{1} / \sigma_{t}\right)} \equiv 0 \text { on } F\right),
\end{aligned}
$$

where in the penultimate equation we have used translation invariance of $\eta_{s}^{\Phi(-x)}$ in space and of the dual in time. Letting $t \rightarrow \infty$ now proves the result when $d \geq 3$. The proof for $d \leq 2$ is the same but easier, since the number of particles remaining at time $\sqrt{t}$ will be I with high probability.

\section{Proof of Theorem 2}

If $R_{t}$ is as above, duality implies

$$
P\left(n_{t}(x, i)=1=P_{x}\left(R_{t}^{1}<0\right),\right.
$$

so the central limit theorem tells us that if $x_{t} / \sqrt{t} \rightarrow y$ then

$$
P\left(\eta_{i}\left(x_{t}, i\right)=1\right) \rightarrow \Phi\left(-y / \sigma_{1}\right) \text {. }
$$

Since the normal distribution function is continuous, the last conclusion implies that $P\left(\eta_{t}(y \sqrt{t}, i)=1\right)$ converges to $\Phi\left(-y_{1} / \sigma_{1}\right)$ uniformly on compact sets. Letting

$$
W=\frac{1}{2 t} \sum_{x \in \mathbb{Z}^{2}} \sum_{i=1}^{2} \eta_{h}(x, i) h(x / \sqrt{t}),
$$

it follows easily that if $h$ is a continuous function with compact support then

$$
\mathrm{E} W \rightarrow \int h(x) \Phi\left(-x / \sigma_{1}\right) \mathrm{d} x
$$

To prove the result now it suffices to show that

$$
\mathrm{E} W^{2} \rightarrow\left(\int h(y) \Phi\left(-y / \sigma_{1}\right) \mathrm{d} y\right)^{2}
$$


for this implies that the variance of $W$ tends to 0 . Writing

$$
\begin{aligned}
\mathrm{E} W^{2} & =\frac{1}{(2 t)^{2}} \sum_{x \in \mathbb{Z}^{2}} \sum_{i=1}^{2} \sum_{y \in \mathbf{Z}^{2}} \sum_{j=1}^{2} h(x / \sqrt{t}) h(y / \sqrt{t}) \mathrm{E}\left\{\eta_{t}(x, i) \eta_{t}(y, j)\right\}\left(\int h(x) \Phi\left(-x / \sigma_{1}\right) \mathrm{d} x\right)^{2} \\
& =\iint h(x) h(y) \Phi\left(-x / \sigma_{1}\right) \Phi\left(-y / \sigma_{1}\right) \mathrm{d} y \mathrm{~d} x
\end{aligned}
$$

we see that to prove (2.2) it suffices to show that if $x_{t} / \sqrt{t} \rightarrow x$ and $y_{t} / \sqrt{t} \rightarrow y$ with $x \neq y$ in the support of $h$ then

$$
P\left(\eta_{h}\left(x_{i}, i\right)=1, \eta_{t}\left(y_{t}, i\right)=1\right) \rightarrow \Phi\left(-x_{1} / \sigma_{1}\right) \Phi\left(-y_{1} / \sigma_{1}\right) .
$$

To prove (2.3) we observe that well-known results about two-dimensional random walk imply that with probability approaching 1 , two random walks started at $x_{t}$ and $y_{t}$ will not hit by time $t$.

\section{Proof of Theorem 3}

Let $A_{t}=\left\{S_{t}^{(0,1), t} \neq S_{t}^{(0,2), t}\right\}$ and $R_{t}^{(0, i), t}$ be the $\mathbb{Z}^{d}$ component of $S_{t}^{(0, i), t}$. The desired result follows from the following facts:

$$
\begin{aligned}
P\left(A_{t}\right) & \sim C / \log t \text { as } t \rightarrow \infty ; \\
\left(R_{t}^{(0,1), t} / \sqrt{t}, R_{t}^{(0,2), t} / \sqrt{t} \mid A_{t}\right) & \Rightarrow\left(Z^{1}, Z^{2}\right)
\end{aligned}
$$

where $Z^{i}$ are independent bivariate normals with covariance matrix $\Gamma$ equal to that of $q$.

To prove these results it is convenient to forget about working backwards in time and to clean up the superscripts. Let $S_{f}^{i}, i=1,2$, have the same distribution as the dual processes starting from $(0, i)$, and let $R_{i}^{i}$ and $Q_{t}^{i}$ be the $\mathbb{Z}^{2}$ and $\{1,2\}$ components of the walks. Let $T_{0}$ be the time of the first jump of the $S_{i}^{i}$; let

$$
\begin{gathered}
T=\inf \left\{t>T_{0}: R_{t}^{1}=R_{t}^{2}\right\} \\
\tau=\inf \left\{t>0: S_{t}^{1}=S_{t}^{2}\right\} .
\end{gathered}
$$

We first address ourselves to (2.4). For random walks on $\mathbb{Z}^{2}$ it is 'well known' that $P(T>t) \sim C / \log t$. We have put 'well known' in quotation marks since it is not so easy to find this result in the library. It can be proved using generating functions and Tauberian theorems as in Kesten and Spitzer (1963, pp. 305-306), or one can find a more refined result about $P(T=n)$ in Theorem 4.1 of Jain and Pruitt (1970). However, we have found a very simple argument, which for completeness we will give here.

We begin by considering a discrete-time walk $W_{n}$. Let $T=\inf \left\{m>0: W_{m}=0\right\}, a_{n}=$ $P_{0}(T>n), p_{n}=P_{0}\left(W_{n}=0\right)$ and $g_{n}=\sum_{m=0}^{n} p_{m}$. By considering the last visit to 0 we have $1=\sum_{m=0}^{n} p_{m} a_{n-m}$, and since the $a_{m}$ are monotone it follows that $a_{n} \leq g_{n}^{-1}$. To reverse the last inequality, we note that the invariance principle implies that the probability of a visit to 0 between times $n$ and $2 n$ goes to 0 , so if $\epsilon>0$ and $n$ is large,

$$
1-\epsilon \leq \sum_{m=0}^{n} p_{m} a_{2 n-m} \leq a_{n} g_{n}
$$


Combining this with the previous result shows $a_{n} g_{n} \rightarrow 1$. The local central limit theorem implies $p_{n} \sim C / n$, so $g_{n} \sim C / \log n$ and the desired result follows.

To pass from discrete time to a continuous-time random walk that jumps at rate $\lambda$ is trivial since well-known large deviations results for the Poisson distribution - see, for example, Durrett (1991, Section 1.9) - imply that the probability of more than $(\lambda+\epsilon) t$ or fewer than $(\lambda-\epsilon) t$ jumps by time $t$ is smaller than $\mathrm{Ce}^{-\gamma t}$ for some $0<\gamma, C<\infty$. To extend the conclusion to our random walks on $\mathbb{Z}^{2} \times\{1,2\}$, note that after $T_{0}, R_{t}^{i}$ and $Q_{t}^{i}$ are independent, so even if we condition on the paths $R_{t}^{1}$ and $R_{t}^{2}$ for $t \leq T$ the probability that $\tau=T$ is $1 / 2$. Thus $\tau$ corresponds to the sum of a mean 2 geometric number of returns with distribution $T$. If $T_{1}, \ldots, T_{m}$ are independent and have the same distribution as $T$ then

$$
\begin{aligned}
P\left(T_{1}+\ldots+T_{m}>t\right) & \leq m P(T>t / m) \\
P\left(T_{1}+\ldots+T_{m}>t\right) & \geq P\left(T_{i}>t \text { for some } i\right) \\
& \geq m P(T>t)-\left(\begin{array}{c}
m \\
2
\end{array}\right) P(T>t)^{2} .
\end{aligned}
$$

Multiplying by $2^{-m}$ and summing gives

$$
2 P(T>t)-C P(T>t)^{2} \leq P(\tau>t) \leq 2 P(T>t / \log t)+\sum_{m>\log t} m 2^{-m}
$$

which proves (2.4) since the last term goes to 0 as $t^{-\epsilon}$ for some $\epsilon>0$.

We now tum to (2.5). Since this is more complicated we will give the details first for $\mathbb{Z}^{2}$ and then indicate the generalization to $\mathbb{Z}^{2} \times\{1,2\}$. Let $V_{l}^{1}$ and $V_{l}^{2}$ be independent random walks that make jumps according to $q$ at rate 1 , and let $\tau=\inf \left\{t: V_{t}^{1}=V_{t}^{2}\right\}$. We will work with characteristic functions. Let

$$
\left.\phi_{i}(\lambda)=\mathrm{E}\left[\exp \left\{\mathrm{i}\left(\lambda \cdot V_{t}^{j}\right)\right\}\right]=\exp [-t\{1-\phi(\lambda))\}\right]
$$

where $\phi(\lambda)=\sum_{x} \exp (\mathrm{i} \lambda \cdot x) q(x)$. For future reference, note that

$$
1-\phi(\lambda) \sim \frac{1}{2} \sum_{i j} \lambda_{i} \Gamma_{i j} \lambda_{j}, \quad \text { as } \lambda \rightarrow 0,
$$

where $\Gamma_{i j}=\sum_{x} x_{i} x_{j} q(x)$ for $1 \leq i, j \leq 2$. To investigate the joint distribution of $\left(V_{t}^{1}, V_{t}^{2}\right)$, we fix $\lambda$, $\mu \in \mathbb{R}^{2}$, and for convenience write $W_{t}=\lambda \cdot V_{t}^{1}+\mu \cdot V_{t}^{2}$. Since the two random walks are independent

$$
\mathrm{E}\left\{\exp \left(\mathrm{i} W_{t} / \sqrt{t}\right)\right\}=\phi_{t}\left(\frac{\lambda}{\sqrt{t}}\right) \phi_{t}\left(\frac{\mu}{\sqrt{t}}\right) .
$$

Fix $1 / 2<\alpha<\beta<1, \alpha$ close to 1 , let $0<\epsilon<\min \{\beta-\alpha, 1-\beta\}$, and note that

$$
\left|\mathrm{E}\left\{\exp \left(\mathrm{i} W_{t} / \sqrt{t}\right) ; \tau \in\left(t^{\alpha}, t\right]\right\}\right| \leq P\left(\tau \in\left(t^{\alpha}, t\right]\right) .
$$

To complete the proof now it suffices to show

$$
\mathrm{E}\left\{\exp \left(\mathrm{i} W_{t} / \sqrt{t}\right) ; \tau \leq t^{\alpha}\right\}=\phi_{t}\left(\frac{\lambda}{\sqrt{t}}\right) \phi_{t}\left(\frac{\mu}{\sqrt{t}}\right) P\left(\tau \leq t^{\alpha}\right)+o\left(t^{-\tau}\right)
$$


since combining (2.9) with (2.7) and (2.8) gives

$$
\left|\mathrm{E}\left\{\exp \left(\mathrm{i} W_{t} / \sqrt{t}\right) ; \tau>t\right\}-\phi_{t}\left(\frac{\lambda}{\sqrt{t}}\right) \phi_{t}\left(\frac{\mu}{\sqrt{t}}\right) P\left(\tau>t^{\alpha}\right)\right| \leq P\left(\tau \in\left(t^{\alpha}, t\right]\right)+o\left(t^{-\epsilon}\right) .
$$

Using (2.4), we now get

$$
\limsup _{t \rightarrow \infty}\left|\mathrm{E}\left\{\exp \left(\mathrm{i} W_{t} / \sqrt{t}\right) \mid \tau>t\right\}-\alpha^{-1} \phi_{t}\left(\frac{\lambda}{\sqrt{t}}\right) \phi_{t}\left(\frac{\mu}{\sqrt{t}}\right)\right| \leq \alpha^{-1}-1,
$$

which together with the ordinary central limit theorem is enough to prove the result.

To prove (2.9) now we begin by observing that Kolomogorov's $L^{2}$ maximal inequality (see Durrett 1991, p. 216) implies

$$
P\left(\tau \leq t^{\alpha},\left|V_{\tau}^{i}\right|>t^{\beta / 2}\right) \leq P\left(\sup _{s \leq f^{a}}\left|V_{s}^{i}\right|>t^{\beta / 2}\right) \leq C t^{\alpha-\beta}
$$

Now, making an error of at most $t^{-\varepsilon}$, the left-hand side of (2.9) is

$$
\int_{0}^{t^{\circ}} \sum_{|x| \leq t^{\beta / 2}} P\left(V_{s}^{i}=x, \tau \in \mathrm{d} s\right) \exp \left(\frac{\mathrm{i} x \cdot(\lambda+\mu)}{\sqrt{t}}\right) \phi_{t-s}\left(\frac{\lambda}{\sqrt{t}}\right) \phi_{t-s}\left(\frac{\mu}{\sqrt{t}}\right) .
$$

It is easy to see, since $|x| \leq t^{\beta / 2}$ and $s \leq t^{\alpha}$, that

$$
\exp \left(\frac{\mathrm{i} x \cdot(\lambda+\mu)}{\sqrt{t}}\right)=1+o\left(t^{-\epsilon}\right)
$$

and, recalling $\phi_{t}(\lambda)=\exp (-t\{1-\phi(\lambda)\})$, that

$$
\phi_{t-s}\left(\frac{\lambda}{\sqrt{t}}\right) \phi_{t-s}\left(\frac{\mu}{\sqrt{t}}\right)=\phi_{t}\left(\frac{\lambda}{\sqrt{t}}\right) \phi_{t}\left(\frac{\mu}{\sqrt{t}}\right)+o\left(t^{-\epsilon}\right)
$$

Combining the last four equations, the proof for $\mathbb{Z}^{2}$ is complete.

To obtain the result on $\mathbb{Z}^{2} \times\{1,2\}$, we let $\vec{S}_{t}^{j}$ be modified versions of $S_{t}^{j}$ in which the genes pick independent parents even when $\bar{S}_{t}^{3}=\bar{S}_{t}^{2}$. As before, we let $\tilde{R}_{t}^{j}$ and $\bar{Q}_{t}^{j}$ be the $\mathbb{Z}^{2}$ and $\{1,2\}$ components of our walks. Our first step is to prove that if we let $\bar{W}_{t}=\lambda \cdot \hat{R}_{t}^{1}+\mu \cdot \bar{R}_{t}^{2}$ then

$$
\mathrm{E}\left\{\exp \left(\mathrm{i} \bar{W}_{t} / \sqrt{t}\right)\right\}=\phi_{r}(\lambda / \sqrt{t}) \phi_{t}(\mu / \sqrt{t})+o\left(t^{-\epsilon}\right) .
$$

Intuitively this is true because $\bar{R}_{t}^{1}$ and $\bar{R}_{t}^{2}$ move independently except when $\bar{R}_{t}^{1}=\bar{R}_{t}^{2}$, and the total amount of time the equality holds is $O(\log t)$. To argue this formally, we let $V_{t}^{1}=\bar{R}_{t}^{1}$ and $V_{t}^{2}$ be a process that takes the same jumps as $\bar{R}_{t}^{2}$ when $\bar{R}_{t}^{2} \neq \bar{R}_{t}^{1}$ and takes independent jumps when $\bar{R}_{t}^{2}=\bar{R}_{t}^{1}$.

We can now proceed to estimate the difference as in Durrett and Neuhauser (1994) or Durrett and Swindle (1994). To estimate the amount of time that $\tilde{R}_{t}^{2}=\vec{R}_{t}^{1}$, we note that $\Delta_{t}=\vec{R}_{t}^{2}=\vec{R}_{t}^{1}$ is a modified random walk that takes jumps according to $q$ at rate 2 when $\Delta_{t} \neq 0$, and jumps according to the convolution $q * q$ at rate 1 when $\Delta_{t}=0$. Let $p=(q * q)(0)$. Once we have $\Delta_{t}=0$, this situation will persist for a geometric number of jumps with mean $1 / p$ and then we will have $\Delta_{s} \neq 0$. Once $\Delta_{s} \neq 0$ it follows from the random walk analogue of (2.4) that there is probability of $C / \log t$ or greater of not having $\Delta_{s}=0$ again before time $t$. 
From the last estimate it follows that the probability $Z_{t}=\left\{s \leq t: \Delta_{s}=0\right\}$ will consist of more than $t^{\epsilon / 2}$ intervals is smaller than

$$
(1-C / \log t)^{t / 2} \leq \exp \left(-C t^{t / 2} / \log t\right) .
$$

It is easy to see that with probability greater than or equal to $1-o\left(t^{-\epsilon}\right)$ none of the geometric $(p)$ waiting times or of the exponential holding times will be larger than $C \log t$. Thus with high probability the total amount of time $\left|Z_{t}\right| \leq C t^{t / 2}(\log t)^{2} \leq C t^{t}$. Using Kolmogorov's inequality, it follows that

$$
P\left(\sup _{s \leq t}\left|\bar{R}_{s}^{2}-V_{s}^{2}\right| \geq t^{\epsilon}\right) \leq C t^{\epsilon} / t^{2 \epsilon}+o\left(t^{-\varepsilon}\right) .
$$

Using the last result and the fact that $(2.7)$ holds for the $V_{i}^{i},\left(2.7^{\prime}\right)$ follows easily. It is now routine to extend (2.9) to $\bar{W}_{t}$. Since (2.8) and (2.4) hold, we have extended the proof to $\mathbb{Z}^{2} \times\{1,2\}$.

\section{Random walk estimates}

In this section we will prove the random walk estimates that are needed for the proof of Theorem 4. It may be wise to read Section 4 first to see how Lemmas 2-4 are used before plunging into these details. Many of the results here are minor variations of classical random walk results that we were not able to find in the literature. However, the first result seems to be of independent interest. Let $S_{n}=X_{1}+\ldots+X_{n}$ be a one-dimensional aperiodic random walk. Let $T_{y}=\inf \left\{n: S_{n}=y\right\}$ and let

$$
a(x)=\sum_{m=0}^{\infty} P^{0}\left(S_{m}=0\right)-P^{0}\left(S_{m}=x\right)
$$

be the recurrent potential kernel. Let $\tau_{0}=\inf \left\{n: S_{n} \leq 0\right\}$.

Theorem 8 If $\mathrm{E} X_{i}=0$ and $\mathrm{E} X_{i}^{2}=\sigma^{2} \in(0, \infty)$, then, for $x \geq 0$,

$$
a(x)=x / \sigma^{2}+E^{x}\left\{\left(S_{\tau_{0}} / \sigma^{2}\right)+a\left(S_{\tau_{0}}\right)\right\},
$$

and hence if $\mathrm{E}\left|X_{i}\right|^{3}<\infty$, then

$$
a(x)=x / \sigma^{2}+\alpha+o(1)
$$

\section{Remark}

We have stated the result in discrete time since that is the classical setting for random walk. Since the proof only uses the optional stopping theorem it should be clear that the result is also valid in continuous time.

\section{Proof}

Equation (3.2) is a well-known consequence of (3.1). (See Theorem 3 of Lai (1976) for a proof for the more difficult non-lattice case.) To prove (3.1), we begin with the following technical result. $4 T$ is the time $S_{n}$ first exits from $(0, N-1)$

$$
\mathrm{E}^{x}\left(\left|S_{T}\right| ; S_{T} \leq 0\right) \leq C_{x} \mathrm{E} X_{1}^{2},
$$


where $C_{x}=1 / P_{0}\left(T_{x}<T_{0}\right)$. We prove this result as follows. Let $V_{0}(k)$ be the number of visits to $k$ before hitting 0 and observe that

$$
P^{x}\left(\left|S_{T}\right| \geq \ell ; S_{T} \leq 0\right) \leq \sum_{k=1}^{N-1} \mathrm{E}^{x} V_{0}(k) P\left(\left|X_{1}\right| \geq \ell+k\right)
$$

To bound the right-hand side note that $1=\mathrm{E}^{0}\left\{V_{0}(k)\right\} \geq P^{0}\left(T_{x}<T_{0}\right) \mathrm{E}^{x}\left\{V_{0}(k)\right\}$ so $\mathrm{E}^{x} V_{0}(k) \leq C_{x}$ and

$$
\begin{aligned}
\mathrm{E}^{x}\left(\left|S_{T}\right| ; S_{T} \leq 0\right) & =\sum_{\ell=1}^{\infty} P^{x}\left(\left|S_{T}\right| \geq \ell ; S_{T} \leq 0\right) \\
& \leq \sum_{\ell=1}^{\infty} \sum_{k=1}^{N-1} C_{x} P\left(\left|X_{1}\right| \geq \ell+k\right) \\
& \leq \sum_{m=1}^{\infty} C_{x} m P\left(x_{1} \geq m\right) \leq C_{x} \mathrm{E} X_{1}^{2}<\infty
\end{aligned}
$$

thus (3.3) is proved.

Observing that $a(x)$ is a harmonic function for the random walk stopped at 0 , and using the optional stopping theorem at $T$ we have

$$
a(x)=\mathrm{E}^{x}\left\{a\left(S_{T}\right) ; S_{T} \leq 0\right\}+\mathrm{E}^{x}\left\{a\left(S_{T}\right) ; S_{T} \geq N\right\} .
$$

To see that this is justified, replace $T$ by $T \wedge n$, recall $a(x) \geq 0$, let $n \rightarrow \infty$, and use the monotone convergence theorem and the easy fact that $\mathrm{E}_{x}\left(a\left(S_{n}\right) ; T>n\right) \rightarrow 0$.

Since $S_{n}$ is a martingale, using the optional stopping theorem at time $T$ gives

$$
x=\mathrm{E}^{x}\left(S_{T} ; S_{T} \leq 0\right)+\mathrm{E}^{x}\left(S_{T} ; S_{T} \geq N\right) .
$$

To argue that this is valid, replace $T$ by $T \wedge n$ and let $n \rightarrow \infty$, using (3.3) and the dominated convergence theorem for the first term, the monotone convergence theorem for the second and the easy fact that $\mathrm{E}^{x}\left(S_{n} ; T>n\right) \rightarrow 0$. Using (3.3) again, we now have

$$
\mathrm{E}^{x}\left(S_{T} ; S_{T} \leq 0\right) \leq\left(x+C_{x} \mathrm{E} X_{1}^{2}\right) .
$$

Multiplying (3.5) by $1 / \sigma^{2}$ and subtracting from (3.4) we have

$$
a(x)-x / \sigma^{2}=\mathrm{E}^{x}\left\{a\left(S_{T}\right)-S_{T} / \sigma^{2} ; S_{T} \leq 0\right\}+\mathrm{E}^{x}\left\{a\left(S_{T}\right)-S_{T} / \sigma^{2} ; S_{T} \geq N\right\} .
$$

By monotone convergence the first term converges to $\mathrm{E}^{x}\left(a\left(S_{\tau_{0}}\right)-S_{T_{0}} / \sigma^{2}\right)$, so it suffices to show that the second converges to 0 . To do this we use the fact (Spitzer 1976, Section 29, p. 2) that $a(x) / x \rightarrow \sigma^{2}$, so

$$
\left|a(y)-y / \sigma^{2}\right| \leq \epsilon_{N y} \quad \text { for } y \geq N,
$$

where $\epsilon_{N} \rightarrow 0$ as $N \rightarrow \infty$. Using this and (3.6) we have

$$
\mathrm{E}^{x}\left\{\left|a\left(S_{T}\right)-S_{T} / \sigma^{2}\right| ; S_{T} \geq N\right\} \leq \epsilon_{N} \mathrm{E}^{x}\left(S_{T} ; S_{T} \geq N\right) \leq \epsilon_{N}\left(x+C_{x} \mathrm{E} X_{1}^{2}\right) \rightarrow 0,
$$

and the proof is complete. 
Let $X_{\mathrm{i}}(t), X_{2}(t)$ denote independent continuous-time, rate-one random walks with jump matrix $p(x, y)$, and define

$$
\begin{aligned}
& Y_{1}(t)=X_{1}(t)+X_{2}(t) \\
& Y_{2}(t)=X_{2}(t)-X_{1}(t) .
\end{aligned}
$$

Then $Y(t)=\left(Y_{1}(t), Y_{2}(t)\right)$ is a random walk on $\mathbb{Z}^{2}$, in which each $Y_{i}(t)$ is a rate-two random walk on $\mathbb{Z}$ with jump matrix $p(x, y)$. Here we are using our symmetry assumption, but note that if $p(x, y)$ is general, $Y_{2}(t)$ is symmetric. Let

$$
\begin{gathered}
\tau=\inf \left\{t \geq 0: X_{1}(t)=X_{2}(t)\right\}=\inf \left\{t \geq 0: Y_{2}(t)=0\right\} \\
\tau_{-}=\inf \left\{t \geq 0: X_{1}(t)<X_{2}(t)\right\}=\inf \left\{t \geq 0: Y_{2}(t)<0\right\} .
\end{gathered}
$$

Using $P^{x}$, here and in what follows, for the law of $Y_{2}$ given $Y_{2}(0)=x$, we define the probability of no return to 0 by time $t$ starting from $x$ by

$$
\begin{aligned}
& R_{t}^{x}=P^{x}(\tau>t) \quad x \neq 0 \\
& R_{t}^{0} \simeq \sum_{y} p(0, y) P^{y}(\tau>t) .
\end{aligned}
$$

The definition for $x=0$ is chosen to be useful in a last time at 0 decomposition. In what follows, readers (and authors) will have to remember that the case $x=0$ is exceptional.

We will also be interested in the transition probability $p_{i}(x, y)$ and recurrent potential kernel $a(x)$ for $Y_{2}$, defined by

$$
\begin{aligned}
p_{t}(x, y) & =P^{x}\left(Y_{2}(t)=y\right) \\
a(x) & =\int_{0}^{\infty}\left(p_{t}(0,0)-p_{t}(0, x)\right) \mathrm{d} t .
\end{aligned}
$$

We being with an estimate for the first quantity:

$$
0 \leq p_{t}(0,0)-p_{t}(0, x) \leq C x^{2} / t^{3 / 2} .
$$

We can establish this result as follows. If $\phi$ is the characteristic function of $p(0, x)$, then $p_{t}(0, x)$ has characteristic function

$$
\sum_{n=0}^{\infty} \mathrm{e}^{-2 t} \frac{(2 t)^{n}}{n !} \phi^{n}=\exp \{-2 t(1-\phi)\}
$$

so by the inversion formula

$$
\begin{aligned}
p_{t}(0, x) & =\frac{1}{2 \pi} \int_{-\pi}^{\pi} \exp (-\mathrm{i} x \lambda) \exp \{-2 t(1-\phi(\lambda)\} \mathrm{d} \lambda \\
& =\frac{1}{2 \pi} \int_{-\pi}^{\pi} \cos (x \lambda) \exp \{-2 t(1-\phi(\lambda)\} \mathrm{d} \lambda
\end{aligned}
$$


since $\phi(\lambda)$ is real and $\cos$ is even. Taking $x=0$ and subtracting the last equation we have

$$
p_{t}(0,0)-p_{t}(0, x)=\frac{1}{2 \pi} \int_{-\pi}^{\pi}(1-\cos (x \lambda)) \exp \{-2 t(1-\phi(\lambda)\} \mathrm{d} \lambda \leq 0 .
$$

To find an upper bound for the right-hand side, we note that irreducibility implies $\phi(\lambda) \neq 1$ when $\lambda \in[-\pi, \pi]-\{0\}$ and finite variance implies

$$
1-\phi(\lambda) \sim \lambda^{2} \sigma^{2} \quad \text { as } \lambda \rightarrow 0 .
$$

So there exists $\epsilon_{0}>0$ such that $1-\phi(\lambda) \geq \epsilon_{0} \lambda^{2} / 2$ for all $\lambda \in[-\pi, \pi]$. Combining the last observation with the inequality $1-\cos x \leq x^{2} / 2$ (which can be proved by integrating $\sin x \leq x$ for $x>0$ ), we have

$$
p_{t}(0,0)-p_{t}(0, x) \leq \frac{1}{2 \pi} \int_{-\pi}^{\pi} \frac{x^{2} \lambda^{2}}{2} \exp \left(-\epsilon_{0} t \lambda^{2}\right) \mathrm{d} \lambda .
$$

Changing variables $\lambda=u / \sqrt{t}$, the last integral is

$$
\leq \frac{x^{2}}{4 \pi t^{3 / 2}} \int_{-\infty}^{\infty} u^{2} \mathrm{e}^{-\varepsilon_{0} t^{2}} \mathrm{~d} u=C x^{2} / t^{3 / 2}
$$

which gives result (3.8).

We turn now to the probabilities of no return:

$$
R_{t}^{0} \sim \sigma / \sqrt{\pi t} \quad \text { as } t \rightarrow \infty .
$$

To get from the discrete-time result (Spitzer 1976, Section 32, p. 3) to formula (3.9), we begin by noting that if $J_{0}$ is the time of the first jump then $R_{i}^{0}$ is the probability of no return to 0 between times $J_{0}$ and $J_{0}+t$. Next, we observe that well-known large-deviations results for the Poisson distribution (see, for example, Durrett 1991, Section 1.9) imply that the probability of more than $(2+\epsilon) t$ or less than $(2-\epsilon) t$ jumps by time $J_{0}+t$ is smaller than $C \mathrm{e}^{-\gamma t}$ for some $0<\gamma, C<\infty$.

For $x \neq 0$, we have the following results as $t \rightarrow \infty$ :

$$
\begin{aligned}
& R_{t}^{x} \leq C|x| \sqrt{t} \\
& R_{t}^{x} \sim \sigma a(x) / \sqrt{\pi t} .
\end{aligned}
$$

To establish these results we proceed as follows. Since $R_{t}^{x} \leq 1$, taking $C \geq 1$ takes care of $x^{2} \geq t$. Assuming now that $|x|^{2} \leq t$ and using a last time at 0 decomposition, we have

$$
\begin{aligned}
P^{x}(\tau \leq t) & =\int_{0}^{t} p_{s}(x, 0) R_{t-s}^{0} \mathrm{~d} s+p_{t}(x, 0) \quad \text { for } x \neq 0 \\
1 & =\int_{0}^{t} p_{s}(0,0) R_{t-s}^{0} \mathrm{~d} s+p_{t}(0,0) .
\end{aligned}
$$

Subtracting (3.11a) from (3.11b),

$$
R_{t}^{x}=P^{x}(\tau>t)=\int_{0}^{s}\left(p_{s}(0,0)-p_{s}(x, 0)\right) R_{t-s}^{0} \mathrm{~d} s+p_{t}(0,0)-p_{t}(x, 0) .
$$


Using symmetry, (3.8) and (3.9) now, we have

$$
\begin{aligned}
R_{t}^{x} \leq & \int_{0}^{t}\left(p_{s}(0,0)-p_{s}(x, 0)\right) \frac{C}{\sqrt{t-s}} \mathrm{~d} s+\frac{C x^{2}}{t^{3 / 2}} \\
\leq & \int_{0}^{t / 2}\left(p_{s}(0,0)-p_{s}(0, x)\right) \frac{C}{\sqrt{t / 2}} \mathrm{~d} s \\
& +\int_{t / 2}^{t}\left(\frac{C x^{2}}{s^{3 / 2}}\right) \frac{C}{\sqrt{t}-s} \mathrm{~d} s+\frac{C x^{2}}{t^{3 / 2}} .
\end{aligned}
$$

Changing variables $s=r t$ in the second integral and then using the assumption $x^{2} \leq t$ and (3.8), the last expression is

$$
\leq \frac{C a(x)}{\sqrt{t}}+\frac{C x^{2}}{t} \int_{1 / 2}^{1} r^{-3 / 2}(1-r)^{-1 / 2} \mathrm{~d} s+\frac{C|x|}{\sqrt{t}} \leq \frac{C|x|}{t^{1 / 2}} .
$$

This proves (3.10a). To prove (3.10b) we now note that if $\epsilon>0$, then

$$
\begin{aligned}
0 & \leq R_{t}^{x}-\int_{0}^{\epsilon t}\left(p_{s}(0,0)-p_{s}(0, x)\right) R_{t-s}^{0} \mathrm{~d} s \\
& \leq \int_{\epsilon t}^{t}\left(\frac{C x^{2}}{s^{3 / 2}}\right) \frac{C}{\sqrt{t-s}} \mathrm{~d} s+\frac{C x^{2}}{t^{3 / 2}} .
\end{aligned}
$$

When multiplied by $\sqrt{t}$ the two terms on the right go to 0 . The desired result now follows from (3.9) and the fact that $\epsilon$ is arbitrary.

Lemma $1 \mathrm{E}^{x}\left\{Y_{2}^{2}(t) ; \tau>t\right\}=x^{2}+\sigma^{2} \int_{0}^{t} R_{s}^{x} \mathrm{~d} s$.

Proof

Since $\mathrm{E}^{x}\left\{Y_{2}^{2}(t)\right\}=x^{2}+\sigma^{2} t$, it suffices to show that

$$
\mathrm{E}^{x}\left\{Y_{2}^{2}(t) ; \tau \leq t\right\}=\sigma^{2} t-\sigma^{2} \int_{0}^{t} R_{s}^{x} \mathrm{~d} s
$$

Decomposing according to the first hitting time of 0 , we have

$$
\begin{aligned}
\mathrm{E}^{x}\left\{Y_{2}^{2}(t) ; \tau \leq t\right\} & =\int_{0}^{t} P^{x}(\tau \in \mathrm{d} s) \mathrm{E}^{0}\left\{Y_{2}^{2}(t-s)\right\}=\sigma^{2} \int_{0}^{t} P^{x}(\tau \in \mathrm{d} s)(t-s) \\
& =\sigma^{2} \int_{0}^{t} \mathrm{~d} u \int_{0}^{s} P^{x}(\tau \in \mathrm{d} s)=\sigma^{2} t-\sigma^{2} \int_{0}^{t} R_{s}^{x} \mathrm{~d} s
\end{aligned}
$$

Lemma $2 \mathrm{E}^{x}\left(\left|Y_{2}(t)\right| ; \tau>t\right) \leq C|x|$.

Proof

The result is trivial for $x=0$ since $P^{0}(\tau=0)=1$. Jensen's inequality implies

$$
\left[\mathrm{E}^{x}\left\{\mid Y_{2}(t) \| \tau>t\right\}\right]^{2} \leq \mathrm{E}^{x}\left\{Y_{2}^{2}(t) \mid \tau>t\right\}=\mathrm{E}^{x}\left\{Y_{2}^{2}(t) ; \tau>t\right\} / R_{t}^{x} .
$$


Multiplying each side by $\left(R_{t}^{x}\right)^{2}$, taking square roots, then using Lemma 3.1, (3.10) and the trivial inequality $R_{t}^{x} \leq 1$, gives

$$
\begin{aligned}
\mathbf{E}^{x}\left\{\left|Y_{2}(t)\right| ; \tau>t\right\} & \leq\left[R_{t}^{x} \mathrm{E}^{x}\left\{Y_{2}^{2}(t) ; \tau>t\right\}\right]^{1 / 2} \\
& =\left\{R_{t}^{x}\left(x^{2}+\sigma^{2} \int_{0}^{t} R_{s}^{x} \mathrm{~d} s\right)\right\}^{1 / 2} \\
& \leq\left(x^{2}+\frac{C|x|}{\sqrt{t}} \cdot \sigma^{2} \int_{0}^{t} \frac{C|x|}{\sqrt{s}} \mathrm{~d} s\right)^{1 / 2} \\
& =\sqrt{1+2 C^{2} \sigma^{2}}|x|
\end{aligned}
$$

Lemma 3 (a) There is constant $C<\infty$ so that $\mathrm{E}^{x}\left\{Y_{2}^{-}(t) ; \tau>t\right\} \leq C$ for all $x>0$ and $t \geq 0$. (b) $\lim _{t \rightarrow \infty} \mathrm{E}^{x}\left(Y_{2}^{-}(t) ; \tau>t\right)=\left(\sigma^{2} / 2\right)\left(a(x)-x / \sigma^{2}\right)$.

Proof

Decomposing according to $\left(\tau_{-}, Y_{2}\left(\tau_{-}\right)\right)$, we obtain using symmetry and Lemma 2

$$
\begin{aligned}
\mathrm{E}^{x}\left\{Y_{2}^{-}(t) ; \tau>t\right\} & =\sum_{y<0} \int_{0}^{t} P^{x}\left(\tau_{-} \in \mathrm{d} s, Y_{2}(s)=y, \tau>s\right) \mathrm{E}^{y}\left\{Y_{2}^{-}(t-s) ; \tau>t-s\right\} \\
& \leq \sum_{y<0} \int_{0}^{t} P^{x}\left(\tau_{-} \in \mathrm{d} s, Y_{2}(s)=y\right) C|y|=C \mathrm{E}^{x}\left\{\left|Y_{2}\left(\tau_{-}\right)\right| ; \tau_{-} \leq t\right\},
\end{aligned}
$$

but it is known (see Theorem 3 in Lai $(1976$, p. 65)) that under a third moment assumption $\mathrm{E}^{x}\left(\left|Y_{2}\left(\tau_{-}\right)\right|\right)$is bounded in $x>0$.

To prove (b), we note that Theorem $B$ in the Appendix implies

$$
\left\{Y_{2}(t) / \sigma \sqrt{t} \mid \tau>t\right\} \Rightarrow\left\{\frac{1}{2}+p(x)\right\} F_{\sqrt{2} V}+\left\{\frac{1}{2}-p(x)\right\} F_{-\sqrt{2} V},
$$

where $p(x)=x /\left(2 \sigma^{2} \bar{a}(x)\right)$ and $\bar{a}(x)=a(x)$ for $x \neq 0$ and $\bar{a}(0)=1$. Using the definition of conditional expectation, Lemma 1 , and (3.10),

$$
\begin{aligned}
\mathrm{E}^{x}\left[\left\{Y_{2}(t) / \sqrt{t}\right\}^{2} \mid \tau>t\right] & =\frac{\mathrm{E}^{x}\left\{Y_{2}^{2}(t) ; \tau>t\right\}}{t P^{x}(\tau>t)}=\frac{x^{2}+\sigma^{2} \int_{0}^{t} R_{s}^{x} \mathrm{~d} s}{t R_{t}^{x}} \\
& \rightarrow \sigma^{2} \int_{0}^{t} s^{-1 / 2} \mathrm{~d} s=2 \sigma^{2}
\end{aligned}
$$

This shows that $\mathrm{E}^{x}\left[\left\{Y_{2}(t) / \sqrt{t}\right\}^{2} \mid \tau>t\right]$ is bounded in $t$. This fact, Theorem $\mathrm{B}$, and the remark before it that the Rayleigh distribution (here represented by the random variable $V$ ) has mean $\sqrt{\pi / 2}$ imply

$$
\mathrm{E}^{x}\left\{Y_{2}^{-}(t) / \sigma \sqrt{t} \mid \tau>t\right\} \Rightarrow\left\{\frac{1}{2}-p(x)\right\} \sqrt{\pi}
$$


From this and (3.10) it follows that (recall $x>0$ )

$$
\mathrm{E}^{x}\left\{Y_{2}(t) ; \tau>t\right\}=\mathrm{E}^{x}\left\{Y_{2}(t) / \sigma \sqrt{t} \mid \tau>t\right\} \sigma \sqrt{t} R_{t}^{x} \rightarrow\left\{\frac{1}{2}-p(x)\right\} \sqrt{\pi} \sigma \cdot \sigma a(x) / \sqrt{\pi} .
$$

Recalling now that $p(x)=x /\left\{2 \sigma^{2} a(x)\right\}$ gives the desired result.

Let $Q=\{(a, b): a \geq 0>b\}, Q^{\prime}=\{(a, b): a<0 \leq b\}$, and

$$
\tilde{Q}=\{(c, d): d<0, d<c \leq-d\} .
$$

In what follows, $P^{x_{1}, x_{2}}$ will denote the law of $\left(X^{1}, X^{2}\right)$ starting from $X^{i}(0)=x_{i}$ or of $\left(Y^{1}, Y^{2}\right)$ starting from $Y^{i}(0)=x_{i}$; the meaning should be clear from context. $P^{y}$ will continue to denote the law of $Y^{2}$ starting from $Y^{2}(0)=y$.

\section{Lemma 4}

$$
\begin{aligned}
& \sum_{z} P^{z, z+x}(X(t) \in Q, \tau>t)=\mathrm{E}^{x}\left\{Y_{2}^{-}(t) ; \tau>t\right\} \\
& \sum_{z} P^{z, z+x}\left(X(t) \in Q^{\prime}, \tau>t\right)=\mathrm{E}^{x}\left\{Y_{2}^{+}(t) ; \tau>t\right\}
\end{aligned}
$$

\section{Proof}

We will prove only the first equation. Since

$$
\begin{aligned}
& Y_{1}(t)=X_{1}(t)+X_{2}(t) \\
& Y_{2}(t)=X_{2}(t)-X_{1}(t),
\end{aligned}
$$

we have $P^{z_{1} z+x}(X(t) \in Q, \tau \leq t)=P^{2 \tau+x, x}(Y(t) \in \tilde{Q}, \tau \leq t)$. By the Markov property,

$$
\begin{aligned}
P^{2 z+x, x}(Y(t) \in \bar{Q}, \tau \leq t)= & \sum_{y} \int_{0}^{t} P^{2 z+x, x}\left(\tau \in \mathrm{d} s, Y_{1}(s)=y\right) \\
& \cdot \sum_{d<0} \sum_{d<c \leq-d} P^{y, 0}\left(Y_{1}(t-s)=c, Y_{2}(t-s)=d\right) .
\end{aligned}
$$

Translation invariance implies

$$
P^{2 z+x, x}\left(\tau \in \mathrm{d} s, Y_{1}(s)=y\right)=P^{0, x}\left(\tau \in \mathrm{d} s, Y_{1}(s)=y-x-2 z\right),
$$

so summing over $z, y, c$ and then $d$,

$$
\begin{gathered}
\sum_{z} P^{2 z+x, x}(Y(t) \in \tilde{Q}, \tau \leq t) \\
=\sum_{y} \int_{0}^{t} P^{0, x}(\tau \in \mathrm{d} s) \sum_{d<0} \sum_{d<c \leq-d} P^{y, 0}\left(Y_{1}(t-s)=c, Y_{2}(t-s)=d\right) \\
=\int_{0}^{t} P^{x}(\tau \in \mathrm{d} s) \sum_{\substack{d<0 \\
d<c \leq-d \\
c+d \text { even }}} P^{0}\left(Y_{2}(t-s)=d\right) \\
=\int_{0}^{t} P^{x}(\tau \in \mathrm{d} s) \sum_{d<0}|d| P^{0}\left(Y_{2}(t-s)=d\right)=\mathrm{E}^{x}\left\{Y_{2}^{-}(t) ; \tau \leq t\right\} .
\end{gathered}
$$

A similar calculation gives $\sum_{z} P^{z, z+x}(X(t) \in Q)=\mathrm{E}^{x}\left(Y_{2}^{-}(t)\right)$, and we are done. 


\section{Proofs of Theorems 4-6}

\section{Proof of Theorem 4}

To prove Theorem 4, it suffices to show the following:

Proposition 1 The family $\left\{r_{t}-\ell_{t}, t \geq 0\right\}$ is stochastically compact.

\section{Proof}

Let $B_{t}=\sum_{x<y} 1\left\{\eta_{t}(x)=0, \eta_{t}(y)=1\right\}$ be the number of 'inversions'. We claim that

$$
-1 \leq r_{t}-\ell_{t} \leq B_{i} \text {. }
$$

To see this, for $r_{t}-\ell_{t}>0$ we write

$$
\begin{aligned}
r_{t}-\ell_{t}= & 1+\sum_{\ell_{t}<z<r_{t}} 1\left\{\eta_{t}(z)=1\right\}+1\left\{\eta_{t}(z)=0\right\} \\
= & 1\left\{\eta_{t}\left(\ell_{l}\right)=0, \eta_{t}\left(r_{t}\right)=1\right\} \\
& \left.+\sum_{\ell_{t}<z<r_{t}} 1\left\{\eta_{t}\left(\ell_{t}\right)=0, \eta_{t}(z)=1\right\}+1\left\{\eta_{t}(z)=0, \eta_{t}\left(r_{t}\right)=1\right)\right\} \\
\leq & B_{i} .
\end{aligned}
$$

To estimate the number of inversions we will use duality. Recall from Section 2 the definition of the random walks $S_{s}^{x, t}$ that trace the origin of the value at $x$ at time $t$ and the dual process $\xi_{s}^{A, t}=\left\{S_{s}^{x, t} ; x \in A\right\}$. To have a 0 at $x$ and a 1 at $y$ with $x<y$ at time $t$ the two random walks $S_{s}^{x, t}$ and $S_{s}^{y, t}$ must 'cross', that is, they must pass each other without hitting and end up with $S_{t}^{x, t} \geq 0>S_{t}^{y, t}$. Let

$$
D_{K}(w, z, t)=\left\{\xi_{K}^{Z, t} \cap[w, z]=\{w, z\}, S_{i-K}^{w, t-K} \geq 0>S_{t-K}^{2, t-K}\right\},
$$

and let

$$
A_{K}(t)=\sum_{w<z} 1_{D_{K}(w, z, t)}
$$

$D_{K}(w, z, t)$ will be 1 if there are particles at $w$ and $z$ at time $K$ in the dual coalescing random walk with no particles in between, and if these particles cross and end up on opposite sides of 0 at time $t$ in the dual. So $A_{K}(t)$ counts the number of crossings that occur after time $K$. The two keys to the proof are:

Lemma $5 \sup _{t>K} \mathrm{E}\left\{A_{K}(t)\right\} \rightarrow 0$ as $K \rightarrow \infty$.

Lemma $6 \mathrm{E}\left\{B_{i} ; A_{K}(t)=0\right\} \leq C \sigma^{2} K$.

Once these are established the result follows since if we fix $\epsilon>0$, then by Lemma 5 we can choose $K<\infty$ such that $P\left(A_{K}(t)>0\right) \leq \epsilon$ for all $t>K$. Using Markov's inequality and Lemma 6 , it 
follows that for all $t>K$,

$$
\begin{aligned}
P\left(r_{t}-\ell_{t}>M\right) & \leq \epsilon+P\left(B_{t}>M, A_{K}(t)=0\right) \\
& \leq \epsilon+\mathrm{E}\left\{B(t) ; A_{K}(t)=0\right\} / M \\
& \leq \epsilon+C \sigma^{2} K / M \leq 2 \epsilon
\end{aligned}
$$

for sufficiently large $M$.

Proof of Lemma 5

Let

$$
\mu_{K}(w, z)=P\left(\xi_{K}^{Z, t} \cap[w, z]=\{w, z\}\right)
$$

and observe that

$$
\epsilon_{K} \equiv \sum_{x>0} \mu_{K}(0, x)=P\left(0 \in \eta_{K}^{\mathbb{Z}}(0)\right) \rightarrow 0 \quad \text { as } K \rightarrow \infty
$$

For $t>K$ we compute using Fubini's theorem and the Markov property:

$$
\begin{aligned}
\mathrm{E}\left\{A_{K}(t)\right\} & =\sum_{w<z} P\left(D_{K}(w, z, t)\right) \\
& =\sum_{w<z} \mu_{K}(w, z) P\left(S_{t-K}^{w, t-K} \geq 0>S_{t-K}^{z, t-K}\right) \\
& =\sum_{x>0} \mu_{K}(0, x) \sum_{w} P\left(S_{t-K}^{w, t-K} \geq 0>S_{t-K}^{w+x}, t-K\right) \\
& =\sum_{x>0} \mu_{K}(0, x) \mathrm{E}^{x}\left\{Y_{2}^{-}(t-K) ; \tau_{2}(0)>t-K\right\} \leq C \epsilon_{K} .
\end{aligned}
$$

The last equality and the final in equality follow from Lemmas 4 and 3, respectively.

\section{Proof of Lemma 6}

We now estimate the size of $B_{t}$ on the event $\left\{A_{K}(t)=0\right\}$.

$$
\begin{aligned}
\mathrm{E}\left\{B_{t} ; A_{K}(t)=0\right\} & =\sum_{x<y} P\left(S_{i}^{x, t} \geq 0>\hat{S}^{y, t} ; A_{K}(t)=0\right) \\
& \leq \sum_{x<y} \sum_{w<z} P\left(S_{K}^{x, t}=z, S_{K}^{y, t}=w\right) P\left(\hat{\eta}_{t-K}^{z, t-K} \geq 0>\hat{\eta}_{t-K}^{w, t-K}\right) \\
& =\sum_{w<z} \nu_{K}(w, z) P\left(S_{t-K}^{z, t-K} \geq 0>S_{t-K}^{w, t-K}\right),
\end{aligned}
$$

where $\nu_{K}(w, z)=\sum_{x<y} P\left(\hat{\eta}_{K}^{x}=z, \hat{\eta}_{K}^{y}=w\right)$ is an upper bound on the probability that two particles that have crossed by time $K$ reside at $z$ and $w$ at time $K$. 
In Section 3 we introduced independent random walks $X_{1}(t)$ and $X_{2}(t)$ with jump kernel $p(x, y)$ and $Y_{2}(t)=X_{2}(t)-X_{1}(t)$. Using that notation, we can estimate

$$
\begin{aligned}
\nu_{X}(w, z) & \leq \sum_{x<y} P^{x_{1} y}\left(X_{1}(K)=z, X_{2}(K)=w\right) \\
& =\sum_{x<y} P^{0,0}\left(X_{1}(K)=z-x, X_{2}(K)=w-y\right) \\
& =P^{0}\left(Y_{2}(t)<w-z\right)
\end{aligned}
$$

Consequently,

$$
\begin{aligned}
\mathrm{E}\left\{B_{i} ; A_{K}(t)=0\right\} & \leq \sum_{w<2} \nu_{K}(w, z) P^{w, z}\left(X_{1}(t-K)<0 \leq X_{2}(t-K), \tau>t-K\right) \\
& =\sum_{w} \sum_{x>0} \nu_{K}(0, x) P^{w, w+x}\left(X_{1}(t-K)<0 \leq X_{2}(t-K), \tau>t-K\right)
\end{aligned}
$$

by translation invariance. Using Lemma 4 , then Lemma 2 and (4.2), the last expression

$$
\begin{aligned}
\mathrm{E}\left\{B_{i} ; A_{K}(t)=0\right\} & =\sum_{x>0} \nu_{K}(0, x) \mathrm{E}^{x}\left\{Y_{2}^{+}(t-K) ; \tau>t-K\right\} \\
& \leq C \sum_{x>0} x P^{0}\left(Y_{2}(K)<-x\right) \leq C \mathrm{E}^{0}\left\{Y_{2}^{2}(K)\right\}=C \sigma^{2} K .
\end{aligned}
$$

\section{Proof of Theorem 5}

By duality and the central limit theorem, if $x_{t}=r \sigma t^{1 / 2}$, then

$$
P\left(\eta_{t}\left(x_{t}\right)=1\right)=P^{x_{r}}\left(X_{1}(t)<0\right)=P^{0}\left(X_{1}(t)<-x_{t}\right) \rightarrow \Phi(-r)=1-\Phi(r) .
$$

Now $\eta_{t}\left(x_{t}\right)=1$ is only possible if $r_{t} \geq x_{t}$, so

$$
P\left(r_{t} \geq x_{t}\right) \geq P\left(\eta_{t}\left(x_{t}\right)=1\right) \text {. }
$$

To get a bound in the other direction, fix $\epsilon>0$ and choose $M$ such that $P\left(r_{t}-\ell_{t} \geq M\right)<\epsilon$ for all $t>0$, and note that $\eta_{t}\left(x_{t}-M=0\right)$ and $r_{t} \geq x_{t}$ is only possible if $r_{t}-\ell_{t} \geq M$, so

$$
P\left(r_{t} \geq x_{t}\right) \leq \epsilon+P\left(\eta_{t}\left(x_{t}-M\right)=1\right) \text {. }
$$

Since $P\left(\eta_{t}\left(x_{t}-M\right)=1\right) \rightarrow 1-\Phi(r)$ and $\epsilon$ is arbitrary, the result follows.

\section{Proof of Theorem 6}

The first step is to observe that, for $x>0$,

$$
\begin{aligned}
\sum_{z} 1\left\{\eta_{t}(z)=0, \eta_{t}(z+x)=1\right\} & =\sum_{\ell_{t} \leq z<r_{t}} 1\left\{\eta_{t}(z)=0, \eta_{t}(z+x)=1\right\} \\
& \leq \sum_{\ell_{1} \leq x<r_{t}} 1\left\{r_{t}-\ell_{t} \geq x\right\}=\left(r_{t}-\ell_{t}\right) 1\left\{r_{t}-\ell_{t} \geq x\right\} .
\end{aligned}
$$


Next, for Lemma 4 we know that

$$
\mathrm{E}\left(\sum_{z} 1\left\{\eta_{t}(z)=0, \eta_{t}(z+x)=1\right\}\right)=\mathrm{E}^{x}\left\{Y_{2}^{-}(t) ; \tau>t\right\},
$$

and the right-hand side converges to $\left(\sigma^{2} / 2\right)\left\{a(x)-x / \sigma^{2}\right\}$ as $t \rightarrow \infty$. Theorem 8 and Lemma 3 imply that if the steps have finite third moment and we exclude the nearest neighbour case then we have, for some $\delta>0$,

$$
\inf _{x>0} \lim _{t \rightarrow \infty} E\left[\left(r_{t}-\ell_{t}\right) 1\left\{r_{t}-\ell_{t} \geq x\right\}\right]>\delta .
$$

The problem now is to show that $\sum_{k \geq x} k \bar{\pi}_{k}$ is also bounded away from zero as $x \rightarrow \infty$, since this will imply the claim by dominated convergence. To do this we will use the fact that the interface is a positive recurrent chain. Let $\iota_{0}$ denote the trivial interface which corresponds to the initial state we choose in the paper. If we start with the interface in the stationary distribution, then we have, for every $t$,

$$
\begin{aligned}
\sum_{k \geq x} k \bar{\pi}_{k} & =\sum_{i} \pi(\iota) \mathrm{E}^{\iota}\left(r_{t}-\ell_{t}\right) 1\left\{r_{t}-\ell_{t} \geq x\right\} \\
& \geq \pi\left(\iota_{0}\right) \mathrm{E}^{\iota_{0}}\left(r_{t}-\ell_{t}\right) 1\left\{r_{t}-\ell_{t} \geq x\right\} .
\end{aligned}
$$

Letting $t \rightarrow \infty$ and using (4.3), we have that the left-hand side above is bounded below by $\pi\left(\omega_{0}\right) \delta$, and we are done.

\section{Acknowledgements}

Both authors were partially supported by the National Science Foundation and by the Army Research Office through the Mathematical Sciences Institute at Cornell University. Ted Cox was supported in addition by a grant from the National Security Agency.

\section{Appendix}

Here we prove the conditioned limit theorem needed in the proof of Lemma 3. Let $Y(t)$ be a ratetwo, continuous-time, one-dimensional random walk with

$$
\begin{aligned}
& \mathrm{E}^{0}[\exp \{\mathrm{i} \lambda Y(t)\}]=\exp [-2 t\{1-\phi(\lambda)\}] \\
& \phi(\lambda)=1-\frac{\sigma^{2} \lambda^{2}}{2}+o\left(\lambda^{2}\right) \quad \text { as } \lambda \rightarrow 0 .
\end{aligned}
$$

Theorem A If $\tau$ is the first return time to 0 , then

$$
\lim _{t \rightarrow \infty} \mathrm{E}^{x}[\exp \{\mathrm{i} \theta Y(t) / \sigma \sqrt{t}\} \mid \tau>t]=\mathrm{e}^{-\theta^{2}} \cdot \frac{\mathrm{i} \theta \sqrt{\pi} x}{\sigma^{2} \bar{a}(x)}+1-\theta^{2} \int_{0}^{1} \frac{\mathrm{e}^{-v \theta^{2}}}{\sqrt{1-v}} \mathrm{~d} v
$$


where $\bar{a}(x)=1$ for $x=0$, and $\bar{a}(x)=a(x)$ for $x \neq 0$.

Proof

By the Markov property,

$$
\begin{aligned}
\mathrm{E}^{x}[\exp \{\mathbf{i} \lambda Y(t)\} ; \tau \leq t] & =\int_{0}^{t} P^{x}(\tau \in \mathrm{d} s) \mathbf{E}^{0}[\exp \{\mathbf{i} \lambda Y(t-s)\}] \mathrm{d} s \\
& =\int_{0}^{t} P^{x}(\tau \in \mathrm{d} s) \exp [-2(t-s)\{1-\phi(\lambda)\}] \mathrm{d} s .
\end{aligned}
$$

Now observe that

$$
\int_{0}^{t-s} \exp [-2 u\{1-\phi(\lambda)\}] \mathrm{d} u=\frac{1-\exp [-2(t-s)\{1-\phi(\lambda)\}]}{2\{1-\phi(\lambda)\}}
$$

and hence

$$
\begin{aligned}
\mathrm{E}^{x}[\exp \{\mathrm{i} \lambda Y(t)\} ; \tau \leq t] & =\int_{0}^{t} P^{x}(\tau \in \mathrm{d} s)\left(1-2\{1-\phi(\lambda)\} \int_{0}^{t-s} \mathrm{e}^{-2 u\{1-\phi(\lambda)\}} \mathrm{d} u\right) \\
& =1-R_{t}^{x}-2\{1-\phi(\lambda)\} \int_{0}^{t} P^{x}(\tau \in \mathrm{d} s) \int_{0}^{t-s} \mathrm{e}^{-2 u\{1-\phi(\lambda)\}} \mathrm{d} u \\
& =\mathrm{I}-R_{t}^{x}-2\{1-\phi(\lambda)\} \int_{0}^{t} \mathrm{e}^{-2 u\{1-\phi(\lambda)\}}\left(1-R_{i-u}^{x}\right) \mathrm{d} u \\
& =\mathrm{e}^{-2 t\{1-\phi(\lambda)\}}-R_{t}^{x}+2\{1-\phi(\lambda)\} \int_{0}^{t} \mathrm{e}^{-2 u\{1-\phi(\lambda)\}} R_{t-u}^{x} \mathrm{~d} u
\end{aligned}
$$

Noticing that $\mathrm{E}^{x}[\exp \{\mathrm{i} \lambda Y(t)\}]=\mathrm{e}^{\mathrm{i} \lambda x} \mathrm{e}^{-2 t(1-\phi(\lambda)\}}$ and changing variables $u=v t$ in the integral, we have

$$
\begin{aligned}
\mathrm{E}^{x}[\exp \{\mathrm{i} \lambda Y(t)\} ; \tau>t]= & \mathrm{e}^{-2 t\{1-\phi(\lambda)\}}\left(\mathrm{e}^{\mathrm{i} \lambda x}-1\right) \\
& +R_{t}^{x}-2\{1-\phi(\lambda)\} \int_{0}^{1} \exp [-2 v t\{1-\phi(\lambda)\}] R_{t-v t}^{x} t \mathrm{~d} v .
\end{aligned}
$$

Setting $\lambda=\theta / \sigma \sqrt{t}$ and dividing by $R_{t}^{x}$, we have

$$
\begin{aligned}
\mathrm{E}^{x}[\exp \{\mathrm{i} \theta Y(t) / \sigma \sqrt{t}\} \mid \tau>t\}= & \mathrm{e}^{-2 t(1-\phi(\theta / \sigma \sqrt{i})\}} \frac{\mathrm{e}^{\mathrm{j} \theta x / \sigma \sqrt{t}}-1}{R_{t}^{x}} \\
& +1-2 t\{1-\phi(\theta / \sigma \sqrt{t})\} \int_{0}^{1} \exp [-2 v t\{1-\phi(\theta \sigma / \sqrt{t})\}] \frac{R_{t-v t}^{x}}{R_{t}^{x}} \mathrm{~d} v
\end{aligned}
$$

As $t \rightarrow \infty, 2 t\{1-\phi(\theta / \sigma \sqrt{t})\} \rightarrow \theta^{2}$ uniformly on compact sets. Using (3.9) and (3.10), we see that $R_{i}^{x} \sim \sigma \bar{a}(x) / \sqrt{\pi t}$ and

$$
\mathrm{e}^{-2 t\{1-\phi(\theta / \sigma \sqrt{t})\}} \frac{\mathrm{e}^{\mathrm{i} \theta x / \sigma \sqrt{t}}-1}{R_{t}^{x}} \rightarrow \mathrm{e}^{-\theta^{2}} \cdot \frac{\mathrm{i} \theta x / \sigma}{\sigma a(x) / \sqrt{\pi}}
$$


We claim that the final integral in (A.3) converges to

$$
-\theta^{2} \int_{0}^{1} \mathrm{e}^{-2 \theta^{2}} \frac{1}{\sqrt{1-v}} \mathrm{~d} v
$$

To prove this, consider separately the integrals over $\left[0,1-t^{-3 / 4}\right]$ and $\left[1-t^{-3 / 4}, 1\right]$. On the second interval the integrand is bounded by $C \sqrt{t}$ and so the integral is bounded above by $C t^{-1 / 4} \rightarrow 0$. On the first interval the integrands converge to the limiting integrand and are bounded by $2 / \sqrt{1-v}$ for large $t$, so the result follows from the dominated convergence theorem.

Our next step is to invert the characteristic function in Theorem A. Let $V$ be a random variable with the Rayleigh density, that is,

$$
P(V=v)= \begin{cases}v \mathrm{e}^{-v^{2} / 2} & \text { for } v \geq 0 \\ 0 & \text { otherwise }\end{cases}
$$

and for future reference note that recalling the variance of the standard normal shows $\mathrm{E} V=\sqrt{2 \pi} / 2$. If we let $F_{X}$ denote the distribution function of $X$, then we have the following theorem:

Theorem B As $t \rightarrow \infty$,

$$
\{Y(t) / \sigma \sqrt{t} \mid \tau>t\} \Rightarrow\left\{\frac{1}{2}+p(x)\right\} F_{\sqrt{2} V}+\left\{\frac{1}{2}-p(x)\right\} F_{-\sqrt{2} V}
$$

where $p(x)=x /\left\{2 \sigma^{2} \bar{a}(x)\right\}$.

Proof

Integrating by parts and then consulting Belkin $(1970$, p. 157) for the final equality, we have

$$
\begin{aligned}
\frac{1}{2} \int_{-\infty}^{\infty}|x| \mathrm{e}^{-x^{2} / 2} \mathrm{e}^{\mathrm{i} t x} \mathrm{~d} x & =\int_{0}^{\infty} x \mathrm{e}^{-x^{2} / 2} \cos (t x) \mathrm{d} x \\
& =-\left.\mathrm{e}^{-x^{2} / 2} \cos (t x)\right|_{0} ^{\infty}-\int_{0}^{\infty} \mathrm{e}^{-x^{2} / 2} t \sin (t x) \mathrm{d} x \\
& =1-t \int_{0}^{\infty} \mathrm{e}^{-x^{2} / 2} \sin (t x) \mathrm{d} x \\
& =1-\frac{t^{2}}{2} \int_{0}^{1} y^{-1 / 2} \exp \left\{-t^{2}(1-y) / 2\right\} \mathrm{d} y .
\end{aligned}
$$

To check this strange identity at home, let $g_{i}(t)$ denote the last two lines, let $h_{i}(t)=\left\{1-g_{i}(t)\right\} / t$, and note that $h_{i}^{\prime}(t)=1-t h_{i}(t)$, with $h_{i}(0)=0$.

Changing variables $y=1-v, t=\theta \sqrt{2}$, we see that if $U$ has distribution $\left(F_{V}+F_{-V}\right) / 2$, then

$$
\mathrm{E}\left(\mathrm{e}^{\mathrm{i} \theta \sqrt{2} U}\right)=1-\theta^{2} \int_{0}^{1} \frac{\mathrm{e}^{-\imath \theta^{2}}}{\sqrt{1-v}} \mathrm{~d} v .
$$

Comparing with Theorem A, we see that when $x=0$ the result holds with $p(0)=0$. To get the result 
for $x \neq 0$, we observe that integrating by parts and recalling the characteristic function for the normal show that the signed measure $F_{V}-F_{-V}$ has characteristic function

$$
\int_{-\infty}^{\infty} x \mathrm{e}^{-x^{2} / 2} \mathrm{e}^{\mathrm{i} t x} \mathrm{~d} x=-\mathrm{i} t \sqrt{2 \pi} \int_{-\infty}^{\infty}(2 \pi)^{-1 / 2} \mathrm{e}^{-x^{2} / 2} \mathrm{e}^{\mathrm{i} / x} \mathrm{~d} x=-\mathrm{i} t \sqrt{2 \pi} \mathrm{e}^{-t^{2} / 2}
$$

With the change of variables $t=\theta \sqrt{2}$ and comparing with Theorem $\mathrm{A}$, we get

$$
p(x)=x /\left\{2 \sigma^{2} \bar{a}(x)\right\} .
$$

\section{References}

Belkin, B. (1970) A limit theorem for conditioned recurrent random walk attracted to a stable law. Ann. Math. Statist., 41, 146-163.

Belkin, B. (1972) An invariance principle for conditioned recurrent random walk attracted to a stable law. Z. Wahrscheinwichkeitstheorie Veru. Geb., 21, 45-64.

Bezuidenhout, C. and Grimmett, G. (1990) The critical contact process dies out. Ann. Probab., 19, 984-1009. Bramson, M. and Neuhauser, C. (1992) A cataiytic surface reaction model. J. Comput. Appl. Math., 40, 157161.

Durrett, R. (1978) Conditioned limit theorems for some null recurrent Markov processes. Ann. Probab., 6, 798828.

Durrett, R. (1988) Lecture Notes on Particle Systems and Percolation. Belmont, CA: Wadsworth.

Durrett, R. (1991) Proability: Theory and Examples. Belmont, CA: Wadsworth.

Durrett, R. (1993) Ten Lectures on Particle Systems, St. Flour Lecture Notes, Lecture Notes in Math. To appear. New York: Springer-Verlag.

Durrett, R. and Neuhauser, C. (1994) Particle systems and reaction diffusion equations. Ann. Probab., 22, 289333.

Durrett, R. and Swindle, G. (1994) Coexistence results for catalysts. Probab. Theory Related Fields, 98, 489515.

Ferrari, P. (1992) Shock fluctuations in asymmetric simple exclusion. Probab. Theory Related Fields, 91, 81101.

Ferrari, P. and Ravishankar, K. (1992) Shocks in the asymmetric exclusion automata. Ann. Appl. Probab., 2, 928-941.

Ferrari, P., Kipnis, C. and Saada, E. (1991) Microscopic structure of travelling waves in the asymmetric simple exclusion process. Ann. Probab, 19, 226-244.

Griffeath, D. (1979) Additive and Cancellative Interacting Particle Systems, Lecture Notes in Math. 724. New York: Springer-Verlag.

Harris, T.E. (1972) Nearest neighbor Markov interaction processes on multidimensional lattices. Adv. Math., 9, $66-89$.

Harris, T.E. (1978) Additive set-valued Markov processes and graphical methods. Ann. Probab., 6, 355-378.

Harrison, R.G. (1990) Hybrid zones: windows on evolutionary processes. In D. Futuyama and J. Antonovics (eds), Oxford Surveys in Evolutionary Biology. Oxford: Oxford University Press.

Holley, R.A. and Liggett, T.M. (1975) Ergodic theorems for weakly interacting systems and the voter mode]. Ann. Probab., 3, 643-663.

Hunt, W.G. and Selander, R.K. (1973) Biochemical genetics of hybridization in European house mice. Heredity, 31, 11-33.

Jain, N. and Pruitt, W.E. (1970) The range of random walk. Proc. 6th Berkeley Symp., III, 31-50. 
Kesten, H. and Spitzer, F. (1963) Ratio limit theorems for random walks, I. J. Anal. Math., 11, 285-322.

Lai, T.L. (1976) Asymptotic moments of random walks with applications to ladder variables and renewal theory. Ann. Probab., 4, 51-66.

Liggett, T.M. (1985) Interacting Particle Systems. New York: Springer.

Moore, W.S. and Koenig, W.D. (1986) Comparative reproductive success of yellow-shafted, red-shafted and hybrid flickers across a hybrid zone. The Auk, 103, 42-51.

Mueller, C. and Sowers, R. (1994) Random travelling waves for the KPP equation with noise. Preprint.

Presutti, E. and Spohn, H. (1983) Hydrodynamics for the voter model. Ann. Probab., 4, 867-875.

Spitzer, F. (1976) Principles of Random Walk (2nd edn). New York: Springer.

Tribe, R. (1993) A travelling wave solution to the Kolmogorov equation with noise. Preprint.

Wick, D. (1985) A dynamical phase transition in an infinite particle system. J. Phys., 38, 1015-1025.

Ziff, R.M., Gulari, E. and Barshad, Y. (1986) Kinetic phase transitions in an irreversible surface-reaction model. Phys. Rev. Lett., 56, 2553-2556.

Received March 1994 and revised November 1994 